\title{
SEPARABLE ALGEBRAS AND COVERING SPACES
}

\author{
ANDERS HØST-MADSEN
}

\section{Introduction}

This paper is a contribution to the continued work on understanding the relation between the algebraic structure of a commutative Banach algebra $B$ and the topological structure of its maximal ideal space $\Delta_{B}$. It has attracted considerable interest to find the algebraic-topological invariants of $\Delta_{B}$ directly from $B$, and some nice results in this direction have been obtained (see [20]): $H^{0}\left(\Delta_{B}, \mathrm{Z}\right)$ is the additive group generated by the idempotents of $B$, $H^{1}\left(\Delta_{B}, \mathrm{Z}\right) \cong B^{-1} / \exp B$ (due to Arens and Royden) and $H^{2}\left(\Delta_{B}, \mathrm{Z}\right) \cong \operatorname{Pic} B$ (due to Forster). The next logical step is then of course to characterize $H^{3}\left(\Delta_{B}, \mathrm{Z}\right)$. Some success has been obtained in this direction: already Grothendick observed (more or less) that Tor $H^{3}(X, \mathrm{Z}) \cong \mathrm{Br} C(X)$, the Brauer group of the algebra of continuous functions $X \rightarrow \mathrm{C}$, defined from the central separable [Azumaya] algebra of $C(X)$, and this result was later generalized to arbitrary Banach-algebra by Craw and Ross [2].

Concurrent with this research, there has been a considerable research on the relation between the fundamental group $\pi_{1}\left(\Delta_{B}\right)$ and $B$. Finding $\pi_{1}\left(\Delta_{B}\right)$ is equivalent to finding the covering spaces of $\Delta_{B}$. Childs [1] and Wajnryb [22] showed that there is a category equivalence between the finite-fibered covering spaces of a compact topological space $X$ and the projective separable extensions of $C(X)$. This result was generalized to semisimple, regular $\mathrm{Ba}-$ nach-algebras by Magid [14] and to arbitrary Banach-algebras by Zame [23] and Craw and Ross [2] and even further to topological Waelbrook algebras by Mallios [15]. This is also closely connected to the work on polynomial covering spaces of $X$, see [7] and [8].

The problem with both the results on $H^{3}$ and on $\pi_{1}$ is that some finiteness in the algebras considered prevents us from obtaining all of $H^{3}$ and all of $\pi_{1}$. This problem was overcome for $H^{3}$ by Taylor [21] by considering central separable algebras not required to have a unit. He showed that for $C(X)$ the extended Brauer-group defined from these was all of $H^{3}$. This led us to in-

Received January 25, 1995; in revised form December 15, 1997. 
vestigate the commutative, separable algebras [without unit] in the hope that they would give all the covering spaces of $X$. In this paper it is shown that this is in fact true.

In the first section we define the class of separable algebras and Galoisextensions without unit and obtain some essential structural properties of these. In the second section we relate covering spaces to a certain category of algebra bundles, and in the last section we synthesize the results of section 2 and 3 to obtain the category equivalence between Galois-extensions of $C(X)$ and regular covering spaces of $X$.

\section{Separable algebras}

In the following $R$ will denote a commutative ring with unit. By an algebra $A$ over $R$ we will understand an associative algebra, not necessarily possessing a unit. We will by $A^{*}$ denote the $R$-algebra opposite to $A$ and we define the enveloping algebra of $A$ by $A^{e}=A^{*} \otimes A$ (tensor product with respect to $R$ ). The algebra $A$ is then a $A^{e}$-module by $x \cdot(a \otimes b)=a x b$, and we have an $A^{e}$ module homomorphism $\mu: A^{e} \rightarrow A$ defined by

$$
\mu(a \otimes b)=a b
$$

The homomorphism $\mu$ is called the augmentation of $A$. In case $A$ is commutative, $\mu$ is as well an $R$-algebra homomorphism.

We are now in the position to define a separable algebra. The definition is formally equivalent to the definition for algebras with unit, see, e.g., DeMeyer \& Ingrahim [3]

2.1. Definition. An algebra $A$ over $R$ is called separable if the sequence

$$
0 \rightarrow \operatorname{Ker} \mu \rightarrow A^{e} \stackrel{\mu}{\longrightarrow} A \rightarrow 0
$$

is split exact as a sequence of $A^{\mathrm{e}}$-modules.

Separable algebras can also be characterized as follows

2.2. Proposition. The following are equivalent for any $R$-algebra $A$

1) $A$ is separable.

2) $A^{2}=A$ and $A$ is a projective $A^{e}$-module.

Notice that our definition of a separable algebra is weaker than that of Taylor [21]. Taylor has an extra condition in proposition $4^{2)}$. In our terms Taylor's central separable algebras will be central separable faithful algebras (see definition 2.17).

Many of the theorems for separable algebras with unit can be generalized to separable algebras without unit. However, there are two differences that 
necessitate modification of proofs: there is no seperability idempotent for separable algebras without unit (compare [3], proposition II.1.1), and there is no analogy to the main tool in working with separable algebras with unit: the categorical description of separable algebras ([18], proposition 10.4, [3], II.1.5). Proofs can instead be based on direct tensor product calculations, and are as such quite straightforward. Below, a few theorems will be proven.

If $\left\{A_{\beta}\right\}_{\beta \in B}$ is a family of algebras, $\bigoplus_{\beta \in B} A_{\beta}$ will denote the direct sum of the algebras in the category of algebras [without unit], while if $R$ is a ring and $M$ a cardinal, $R^{(M)}$ will denote the direct sum of $M$ copies of $R$. We now have the following generalization of [18], proposition $10.5 \mathrm{~b}$, with a similar proof

2.3. TheOrem. Let $\left\{A_{\beta}\right\}_{\beta \in B}$ be a family of algebras. Then the direct sum algebra

$$
A=\bigoplus_{\beta \in B} A_{\beta}
$$

is separable if and only if each $A_{\beta}$ is separable.

As the ring $R$ is separable as an algebra over itself, the following corollary follows

2.4. Corollary. Let $R$ be a commutative ring (with unit) and $M$ a set. Then $R^{(M)}$ is a separable $R$-algebra.

The following is a generalization of [3], II.1.7.

2.5. TheOREM. Let $A$ be a separable $R$-algebra and $S$ a commutative $R$-algebra with unit. Then $A \otimes_{R} S$ is a separable $S$-algebra.

Proof. In the following, $A^{S}$ will denote $A \otimes_{R} S$. First notice that we have an $R$-module isomorphism $h:\left(A^{S}\right)^{*} \otimes_{R} A^{S} \rightarrow A^{*} \otimes_{R} A \otimes_{R} S$ defined by

$$
h\left(\left(a_{1} \otimes s_{1}\right) \otimes\left(a_{2} \otimes s_{2}\right)\right)=a_{1} \otimes a_{2} \otimes\left(s_{1} s_{2}\right)
$$

by a direct calculation one sees that $h$ is an $\left(A^{S}\right)^{e}$-module homomorphism.

Now let $\mu$ be the augmentation of $A$ and $\varphi$ a homomorphism that splits $\mu$. We define a homomorphism $\varphi_{S}: A^{S} \rightarrow\left(A^{S}\right)^{*} \otimes A^{S}$ by

$$
\varphi_{S}(a \otimes s)=h^{-1}(\varphi(a) \otimes s)
$$

for $a \in A$ and $s \in S$. Then it is seen by direct calculation that the map $\varphi_{S}$ is an $\left(A^{S}\right)^{e}$-homomorphism which splits $\mu_{S}$.

As $A / A I \cong A \otimes R / I$ for any ideal $I \subseteq R$, we have the following 
2.6. Corollary. Let $A$ be a separable $R$-algebra and $I \subseteq R$ an ideal. Then $A$ / AI is a separable R/I-algebra.

The following is a generalization of [3], II.1.11.

2.7. Theorem. Let $A$ be a separable $R$-algebra and let $I \subseteq A$ be a 2-sided ideal satisfying $I A=I$ or $A I=I$. Then $A / I$ is a separable $R$-algebra.

Proof. Let

$$
\kappa: A^{*} \otimes A \rightarrow(A / I)^{*} \otimes A / I
$$

be the canonical map and let $\varphi: A \rightarrow A^{e}$ be a homomorphism splitting the augmentation $\mu$ of $A$. We define $\tilde{\varphi}: A \rightarrow(A / I)^{*} \otimes A / I$ by

$$
\tilde{\varphi}=\kappa \circ \varphi
$$

By using $I A=I$ or $A I=I$ and $A^{2}=A$, it can easily be seen that $\tilde{\varphi}(I)=0$. Thus, $\varphi$ induces a well-defined map $\varphi_{I}: A / I \rightarrow(A / I)^{*} \otimes A / I$, which is a splitting $(A / I)^{e}$-homomorphism of the augmentation $\mu_{I}$ for $A / I$,

The following theorem is the most important theorem in the theory of commutative separable algebras, and we will apply it numerous times. It is a generalization of the fact that a projective, separable algebra with unit is finitely generated ([3], II.2.1). For algebras without unit we cannot expect a similar result in view of theorem. However we have the following

2.8. Theorem. Let $A$ be a separable commutative $R$-algebra, which is projective as an $R$-module, and let $a \in A$ be arbitrary. Then the ideal $(a)=a A$ is contained in a finitely generated $R$-submodule.

Proof. The proof follows the proof of [3], II.2.1. Let $\mu$ be the augmentation of $A$ and $\varphi$ a map that splits $\mu$. As $A$ is $R$-projective, $A$ has a dual basis $\left\{f_{i}, a_{i}\right\}_{i \in I}$. The functions $(x, y) \rightarrow f_{i}(x) y$ are bilinear from $A \times A \rightarrow A$, so there exist homomorphisms $\phi_{i}: A \otimes A \rightarrow A$ satisfying $\phi_{i}(x \otimes y)=f_{i}(x) y$. Then, for any $u \in A \otimes A$ there is a finite set $I(u)$ so that $\mu(u)=\sum_{i \in I(u)} \phi_{i}(u) a_{i}$.

We may assume that $a$ can be written as $a=b c$. For the separability of $A$ ensures us that $a$ can be written as $a=\sum_{i} b_{i} c_{i}$ and $a A \subseteq \sum_{i} b_{i} c_{i} A$. Thus, if $z \in a A$ then $z=b c y$ for some $y \in A$. We now have

$$
z=b c y=b \cdot(c \otimes y)=b \cdot(y \otimes c)
$$

whence 


$$
\begin{aligned}
z=\mu(\varphi(z)) & =\sum_{i \in I(\varphi(z))} \phi_{i}[\varphi(b)(y \otimes c)] a_{i} \\
& =\sum_{i \in I(\varphi(z))} \phi_{i}[\varphi(b)(c \otimes y)] a_{i}
\end{aligned}
$$

We now assume that $\varphi(b)=\sum_{j=1}^{n} b_{j} \otimes \tilde{b}_{j}$. Then

$$
\phi_{i}[\varphi(b)(c \otimes y)]=\left(\sum_{j=1}^{n} f_{i}\left(b_{j} c\right) \tilde{b}_{j}\right) y
$$

Thus, if $f_{i}\left(b_{j} c\right)=0$ for all $j=1 \ldots n$, then $\phi_{i}(\varphi(z))=0$. We conclude that $I(\varphi(z)) \subseteq I\left(b_{1} c\right) \cup \ldots \cup I\left(b_{n} c\right)$, and as the $b_{j}$ 's and $c$ were only dependent on $a$, the latter set is independent of $y$. Let us call this finite indexing set $J(a)$ : $J(a)=I\left(b_{1} c\right) \cup \ldots \cup I\left(b_{n} c\right)$. We then have

$$
z=\sum_{i \in J(a)} \phi_{i}[\varphi(b)(y \otimes c)] a_{i}=\sum_{i \in J(a)} \sum_{j=1}^{n} f_{i}\left(b_{j} y\right) \tilde{b}_{j} c a_{i}
$$

So the finite set $\left\{\tilde{b}_{j} c a_{i}\right\}_{i \in J(a), j=1 \ldots n}$ generates $a A$ as an $R$-module.

Let $A$ be an algebra. By a modular ideal $I$ of $A$ we will understand an ideal of $A$ such that $A / I$ has a unit. The radical of $A$ is the ideal

$$
\operatorname{Rad} A=\cap\{M \mid M \text { is a maximal modular ideal of } A\}
$$

$A$ is said to be semi-simple if $\operatorname{Rad} A=\{0\}$. If $A$ is an algebra over a field, then by [11], I.10 theorem 1 , the elements of $\operatorname{Rad} A$ are either transcendental or nilpotent. On the other hand, for $A$ commutative every nilpotent element must be in the radical.

In the following, let $F$ denote a field. We will prove that any separable $F$ algebra is semi-simple through a number of lemmas

2.9. Lemma. Let A be a commutative, separable F-algebra. Let a, $x \in A$, and suppose that for some $n \in \mathrm{N}$

$$
a x^{n}=0
$$

Then

$$
a x=0
$$

Proof. It suffices to show, that if $a x^{n}=0$ for $n \geq 2$, then $a x^{n-1}=0$. Let $\varphi$ be a splitting map of the augmentation of $A$ and suppose

$$
\varphi(a)=\sum_{i=1}^{m} a_{i} \otimes \tilde{a}_{i}
$$


By hypothesis, $\varphi(a) \cdot\left(x^{n-1} \otimes x^{n-1}\right)=\varphi\left(a \cdot\left(x^{n-1} \otimes x^{n-1}\right)\right)=0$. Thus

$$
0=\sum_{i=1}^{m} a_{i} x^{n-1} \otimes \tilde{a}_{i} x^{n-1}
$$

There exist a maximal linearly independent set of vectors among $\left\{a_{i} x^{n-1}\right\}_{i=1 . . m}$, and we may assume that it is the first $p$ vectors. Since $F$ is a field, for any $i=1 . . m$ there exists coefficients $\left\{\alpha_{i j}\right\}_{j=1 . . p}$, such that

$$
a_{i} x^{n-1}=\sum_{j=1}^{p} a_{i j} a_{j} x^{n-1}
$$

We then have

$$
\begin{aligned}
0 & =\sum_{i=1}^{m} a_{i} x^{n-1} \otimes \tilde{a}_{i} x^{n-1}=\sum_{i=1}^{m}\left(\left(\sum_{j=1}^{p} \alpha_{i j} a_{j} x^{n-1}\right) \otimes \tilde{a}_{i} x^{n-1}\right) \\
& =\sum_{j=1}^{p}\left(a_{j} x^{n-1} \otimes\left(\sum_{i=1}^{m} \alpha_{i j} \tilde{a}_{i} x^{n-1}\right)\right)
\end{aligned}
$$

As $\left\{a_{j} x^{n-1}\right\}_{j}=1 . . p$ are linearly independent, it follows by standard tensor product rules that

$$
\sum_{i=1}^{m} \alpha_{i j} \tilde{a}_{i} x^{n-1}=0, \quad j=1 . . p
$$

We now can calculate

$$
\begin{aligned}
a x^{n-1} & =\left(\sum_{i=1}^{m} a_{i} \tilde{a}_{i}\right) x^{n-1}=\sum_{j=1}^{p} a_{j}\left(\sum_{i=1}^{m} \alpha_{i j} \tilde{a}_{i} x^{n-1}\right) \\
& =0
\end{aligned}
$$

2.10. Lemma. Let $A$ be a commutative, separable F-algebra. Then $\operatorname{Rad} A$ consists of the nilpotent elements of $A$, and $A \operatorname{Rad} A=\{0\}$.

Proof. By theorem 2.8, there exists no transcendental elements of $A$. Thus, for any $r \in \operatorname{Rad} A$ there exist an $n \in \mathrm{N}$ such that $r^{n}=0$, and hence for any element $a \in A, a r^{n}=0$. By lemma 2.9, $a r=0$, that is $A r=\{0\}$.

(Lemma 2.9 and lemma 2.10 are due to discussions with Michel Solovej and Thomas Vils Petersen).

Notice that every semi-simple, finite-dimensional commutative $F$-algebra $A$ has a unit. To see this, we can adjoin a unit to $A$, which again gives a fi- 
nite-dimensional, semi-simple $F$-algebra $A_{1}$, and use Wedderburn's structure theorem ([18], theorem 3.5) on $A_{1}$. Since $A$ is an ideal of $A_{1}$, the structure of $A_{1}$ shows that $A$ has a unit. We then get

2.11. Lemma. Let $A$ be a semi-simple, commutative F-algebra. If $a \in A$ is contained in a finite dimensional subalgebra, then $a \in a A$.

2.12. Theorem. Any commutative, separable F-algebra A is semisimple.

Proof. Let $r \in \operatorname{Rad} A$, and write $r$ as $r=\sum_{i=1}^{n} b_{i} c_{i}$. By theorem 2.8, there exists a finite dimensional ideal $S$ containing $r, b_{i}$, and $c_{i}, i=1 . . n$. The radical of $S$ is $\operatorname{Rad} S=S \cap \operatorname{Rad} A$, since the elements of the radical are the nilpotent elements. By the remarks above, $S / \operatorname{Rad} S$ has a unit. Let $e \in S$ be a unit modulo $\operatorname{Rad} S$. Then

$$
\begin{aligned}
e r & =\sum_{i=1}^{n}\left(e b_{i}\right) c_{i}=\sum_{i=1}^{n}\left(b_{i}+r_{i}\right) c_{i}=\sum_{i=1}^{n} b_{i} c_{i} \\
& =r
\end{aligned}
$$

where $r_{i}, r_{2}, \ldots, r_{n} \in \operatorname{Rad} S$ and where we have used lemma 2.10. By the same lemma, on the other hand, $e r=0$. Thus, $r=0$.

Theorem 2.12 is not true for non-commutative algebras even if they are finite dimensional, as the following example shows

2.13. Example (Due to A. Ya. Helemskii). Consider the following subalgebra of $M_{2}$ :

$$
\left\{\left(\begin{array}{cc}
\alpha & \beta \\
0 & 0
\end{array}\right) \mid \alpha, \beta \in \mathrm{C}\right\}
$$

This is isomorphic to the 2-dimensional algebra of pairs $(\alpha, \beta)$ with multiplication given by

$$
(\alpha, \beta)(\gamma, \delta)=(\alpha \gamma, \alpha \delta)
$$

This certainly is an algebra, it is not commutative nor semi-simple (the ideal generated by $(0,1)$ is nil), but it is separable. Consider the map $\varphi: A \rightarrow A^{e}$ defined by

$$
(\alpha, \beta) \mapsto(1,0) \otimes(\alpha, \beta)
$$

this map splits the augmentation $\mu: A^{e} \rightarrow A$ and it is also an $A^{e}$-homomorphism, since

$$
\varphi[(\alpha, \beta) \cdot((a, b) \otimes(c, d))]=\varphi[(a \alpha c, a \alpha d)]=(1,0) \otimes(a \alpha c, a \alpha d)
$$

while 


$$
\begin{aligned}
\varphi[(\alpha, \beta)((a, b) \otimes(c, d)) & =((1,0) \otimes(\alpha, \beta))((a, b) \otimes(c, d)) \\
& =(1,0) \otimes(a \alpha c, a \alpha d)
\end{aligned}
$$

We have the following corollary to theorem

2.14. Corollary. Let $R$ be a semi-simple ring (with unit). Then any projective and separable commutative R-algebra is semi-simple.

Proof. Note that, if $P$ is a projective $R$-module,

$$
\left(\bigcap_{M \in \mathscr{N}(R)} M\right) P=\bigcap_{M \in \mathscr{N}(R)}(M P)
$$

where $\mathscr{N}(R)$ denotes the set of maximal ideals in $R$. The inclusion $\subseteq$ is trivial. To prove the inclusion $\supseteq$ let $\left(f_{k}, t_{k}\right)$ be a dual basis of $P$. Let $x \in \bigcap_{M \in \mathscr{N}(R)}(M P)$, and let $M$ be an arbitrary maximal ideal of $R$. Then we can write $x$ as

$$
x=\sum_{j=1}^{n} m_{j} p_{j}, \quad m_{j} \in M, p_{j} \in P
$$

We can also write $x$ by the dual basis

$$
x=\sum_{k} f_{k}(x) t_{k}
$$

Now

$$
f_{k}(x)=f_{k}\left(\sum_{j} m_{j} p_{j}\right)=\sum_{j} m_{j} f_{k}\left(p_{j}\right) \in M
$$

Since $M$ was arbitrary, and $f_{k}(x)$ does not depend on $M, f_{k}(x) \in \bigcap_{M \in \mathcal{N}(R)} M$. Thus, $x \in\left(\bigcap_{M \in \mathscr{N}(R)} M\right) P$.

Now suppose $x \in \operatorname{Rad} A$, and let $M \in \mathscr{N}(R)$ be arbitrary. Then $x \in M A$. For, assume not. Then $f(x) \neq 0$, where $f: A \rightarrow A / M A$ is the natural map. By corollary 2.6 and theorem 2.12, there exists a maximal modular ideal $N \subseteq A / M A$ such that $f(x) \notin N$. Then $x \notin f^{-1}(N)$ and $f^{-1}(N)$ is a maximal modular ideal of $A$, a contradiction. Thus

$$
\bigcap_{M \in \mathscr{N}(A)} M \subseteq \bigcap_{N \in \mathscr{N}(R)} N A=(\operatorname{Rad} R) A
$$

And since by hypothesis $\operatorname{Rad} R=0$, the corollary is proved.

Using theorem 2.8 we are now able to completely describe the structure of the separable commutative algebras over fields, namely 
2.15. TheOREM. Let $F$ be a field and let $A$ be a commutative F-algebra. Then $A$ is separable if and only if

$$
\bigoplus_{\alpha \in \mathscr{A}} F_{\alpha}
$$

where each $F_{\alpha}$ is a (finite dimensional) separable field extension of $F$.

Before proving the theorem we state the following immediate consequence

2.16. Corollary. Let $F$ be a separably closed field and $A$ a separable commutative F-algebra. Then $A \cong F^{(m)}$ for some cardinal $m$.

Proof of THEOREM 2.15. The theorem is already proved in one direction in theorem 2.3 and we will prove the other implication. Let $M \subseteq A$ be a maximal modular ideal. According to theorem $2.12 A$ is semisimple, and according to theorem 2.8 every $m \in M$ is contained in a finite dimensional ideal, so according to lemma 2.11, $m \in m A$. Thus, $M=M A$. According to theorem 2.7, $A$ / $M$ is separable. As $M$ certainly is an $F$-direct summand, $M$ is an $A$-module direct summand according to [21], proposition 1.7, that is $A=M \dot{+} F_{m}$, where $F_{M} \cong A / M$ (as $A$-modules, that is, as $R$-algebras) is a field. Let $\mathscr{N}$ denote the set of maximal modular ideals of $A$, and for $M \in \mathscr{N}$ let $F_{M} \subseteq A$ be the uniquely determined subfield of $A$ corresponding to $M$ via the above direct sum. Notice that $F_{M} \cap F_{N}=\{0\}$ for $M \neq N$, since $F_{M} \cap N$ is an ideal of $F_{M}$, and thus $F_{M} \subseteq N$. We consider the subalgebra $S$ of $A$ generated by the $F_{M}$ 's. As each $F_{M}$ is an $A$-module direct summand, and as $F_{M} \cap F_{N}=\{0\}$ for $M \neq N$,

$$
S=\bigoplus_{M \in \mathscr{N}} F_{M}
$$

as algebras. If we can prove that $S$ is all of $A$, we will be through. The set $A \backslash S$ consists of those elements of $A$ which are outside infinitely many maximal modular ideals (since $A$ is semi-simple), that is, the elements for which the projections $p_{M}: A \rightarrow F_{M}$ are non-zero for infinitely many $M$. But such an element $a$ cannot exist, since then $1_{M} \cdot a \neq 0$ for infinitely many $M$, and consequently, as the set $\left\{1_{M} \mid M \in \mathscr{N}: 1_{M} \cdot a \neq 0\right\}$ is linearly independent, the vector space $\operatorname{span}_{M \in \mathscr{N}}\left\{1_{M} \cdot a\right\}$ is infinite dimensional, contradicting the fact established in theorem 2.8 , that $a A$ is finite dimensional. Thus, $A \backslash S$ is empty.

We will finally define Galois extensions. The definition is a direct generalization to algebras without unit of the definition of a Galois extension in [3], chapter III, and will agree with [3] for algebras with unit and no nontrivial idempotents. As we will only be interested in algebras with no nontrivial idempotents, this poses no restriction. 
2.17. Definition. An $R$-algebra $A$ is called faithful, is $A$ is faithful as an $R$-module and for any maximal ideal $M \subseteq R, M A \neq A$. A commutative, faithful algebra is called an extension of $R$.

To an algebra $A$ without unit we associate an algebra with unit, the algebra $\mathscr{M}(A)$ of multipliers of $A$, see [13]. If $A$ is an $R$-algebra, Aut $A$ will denote the group of $R$-algebra automorphisms of $A$. We can then easily prove

2.18. Proposition. Let $A$ be a commutative R-algebra. We then have a homomorphism $h:$ Aut $A \rightarrow$ Aut $\mathscr{M}(A)$ defined by

$$
h(g)[f]=g \circ f \circ g^{-1}
$$

for $g \in$ Aut $A$ and $f \in \mathscr{M}(A)$, and where the composition is as maps $A \rightarrow A$.

If $A$ is order-free (in particular semi-simple), $h$ is injective and $\operatorname{Im} h$ is exactly those elements of Aut $\mathscr{M}(A)$ satisfying $g A \subseteq A$.

Now, if $A$ is an $R$-algebra and $G$ is any subgroup of Aut $A$, we define

$$
\operatorname{Fix}_{G} A=\{a \in A \mid \forall g \in G: g(a)=a\}
$$

Considering Aut $A$ as a subgroup of Aut $\mathscr{M}(A)$ under the homomorphism of proposition 2.18, we can now define

2.19. Definition. An extension $A$ of $R$. $A$ is called normal if

$$
\operatorname{Fix}_{\operatorname{Aut} A} \mathscr{M}(A)=R
$$

2.20. Definition. A projective separable normal extension of a ring $R$ is called a Galois-extension of $R$. A ring $R$ without non-trivial idempotents is said to be separably closed, if $R$ has no Galois-extensions without non-trivial idempotents.

\section{Projective vector bundles}

In this paragraph we will derive some results on projective $C(X)$-modules, and as a main result that the category of covering spaces is equivalent to a certain category of topological algebra bundles. The results in this section are rather trivial (except perhaps 3.5) and on a level of the initial chapters and basic definitions of books on bundles (such as [6] and [10]). We will therefore at most briefly outline proofs.

In the following $X$ will be a compact topological space, and $C(X)$ the ring of continuous complex valued function.

We will only consider a special class of topological C-vector spaces: spaces with the trivial LCS-topology $([9,12])$. For a vector space $V$, the trivial LCStopology is the finest topology that makes $V$ into a locally convex topologi- 
cal vector space. A basis of neighborhoods of 0 is the family of all absorbing, balanced, convex set, and as a family of seminorms can be taken the family of norms on finite dimensional subspaces.

We will consider (vector) bundles over $X$, defined according to [6], with which we assume the reader is familiar, were the fibers (stalks, in the terminology of [6]) are trivial LCS-spaces. A trivial vector bundle over $X$ is a vector bundle of the form $\xi=\left(X \times V, p_{\xi}, X\right)$, where $V$ is a vector space with the trivial LCS-topology. Here $p_{\xi}$ denotes the natural projection onto $X$, and by $\sigma_{\xi}$ we will denote the natural projection onto $V$.

A basis for a vector bundle $\xi$ is defined naturally as a set of sections $\left\{s_{i}\right\}_{i \in I}$, such that $\left\{s_{i}(x)\right\}_{i \in I}$ is a basis of $\xi_{x}$ for every $x \in X$ and for every section $s \in \Gamma(\xi)$ there exist a finite set $F \subseteq I$ such that $s(x) \in \operatorname{span}_{i \in F}\left\{s_{i}(x)\right\}$ for every $x \in X$. It is then easy to prove that a vector bundle is trivial if and only if it has a basis, and locally trivial ([6], definition 17.1) if and only if it has a local basis.

We will in particular be interested in the following category of vector bundles

3.1. Definition. Let $\xi$ be a trivial vector bundle. A projection in $\xi$ is a morphism $p: \xi \rightarrow \xi$ satisfying $p^{2}=p$. A projective subbundle is the image of a projection. A projective vector bundle is a vector bundle, which is isomorphic to a projective subbundle.

Since the projection mapping $p$ is a retraction in a Hausdorff space, we get

\subsection{Lemma. The image of $p$ is closed.}

It is easy to see that a projective vector bundle is a full bundle, and in fact more is true.

3.3. Proposition. Let $X$ be a compact space and let $\xi$ be a projective vector bundle over $X$. Then if $A \subseteq X$ is a closed set and $s: A \rightarrow \xi$ is a section, then $s$ can be extended as a section to all of $X$.

Proof. As $\xi$ is projective, there exists a trivial vector bundle $\zeta=X \times V$, and morphisms $p: \zeta \rightarrow \xi$ and $\xi \rightarrow \zeta$ with $p q=\operatorname{Id}_{\xi}$. Then $q s: A \rightarrow \zeta$ is a section, that is, $\sigma_{\zeta} q s: A \rightarrow V$ is a continuous function, and as $A$ is compact, it can be extended to a continuous function $t: X \rightarrow V$. Then $\tilde{s}=(x, t(x))$ is a section $X \rightarrow \zeta$ coinciding with $q s$ on $A$, and $p \tilde{s}$ is an extension of $s$.

By using [6], proposition 10.10 (see also proposition 17.2), it is easily seen that the section functor $\Gamma$ is a category equivalence between the category of trivial vector bundles over $X$ and free $C(X)$-modules, and that every free 
$C(X)$-module is of the form $C(X, V)$ for some trivial LCS-space $V$. By noting that $\Gamma$ takes projections to projections, we get

3.4. Theorem. Let $X$ be a compact space. Then $\Gamma$ is a category equivalence from the category of projective vector bundles over $X$ to the projective modules over $C(X)$.

The following is a generalization of Serre-Swan's theorem [19] to infinite dimensional vector bundles

3.5. TheOREM. A locally trivial vector bundle $\xi$ over a compact space $X$ is a projective vector bundle.

Proof. The proof follows [19], modified by the fact that dimensions (into $\mathrm{N})$ and inner products are not well-defined.

As $X$ is compact, there is a finite open covering $\left\{U_{1}, \ldots, U_{n}\right\}$ of $X$, such that $\xi \mid U_{i}$ is trivial. Let $\left\{\chi_{1}, \ldots, \chi_{n}\right\}$ be a partition of unity subordinated to $\left\{U_{1}, \ldots, U_{n}\right\}$ (such that the open sets $V_{i}=\left\{x \in X \mid \chi_{i}(x) \neq 0\right\}$ covers $X$ ), and let $\left\{\omega_{1}, \ldots, \omega_{n}\right\}$ be a partition of unity subordinated to the covering $\left\{V_{1}, \ldots, V_{n}\right\}$.

As $\xi \mid U_{i}$ is trivial, there exist vector spaces $T_{i}$ and isomorphisms $h_{i}: \xi \mid U_{i} \rightarrow U_{i} \times T_{i}$. Let $\zeta=X \times\left(T_{1} \oplus \ldots \oplus T_{n}\right)$ and define $p_{i}: \zeta \rightarrow \xi$ by

$$
p_{i}\left(x,\left(t_{1}, \ldots, t_{n}\right)\right)= \begin{cases}h^{-1}\left(x, \chi_{i}(x)\right) & x \in U_{i} \\ (x, 0) & x \notin U_{i}\end{cases}
$$

$p_{i}$ is continuous, as $\chi_{i}$ is 0 on a neighborhood of $X \backslash U_{i}$. Now define $p: \zeta \rightarrow \xi$ by

$$
p(x, t)=\sum_{i=1}^{n} p_{i}(x, t)
$$

On the other hand define morphisms $q_{i}: \xi \rightarrow X \times T_{i}$ by

$$
q_{i}(x, t)= \begin{cases}h_{i}^{-1}\left(x, \frac{\omega_{i}(x)}{\chi_{i}(x)} t\right) & x \in U_{i} \\ (x, 0) & x \notin U_{i}\end{cases}
$$

$q_{i}$ is continuous, since $\omega_{i}(x) / \chi_{i}(x)$ vanishes on a neighborhood of $X \backslash U_{i}$. We then define $q: \xi \rightarrow \zeta$ as the direct sum of the $q_{i}$ 's. It is then seen by direct calculation that $p(q(x, t))=(x, t)$. Thus $p q=\mathrm{id}_{\xi}$, and $\xi$ is a projective vector bundle.

Serre-Swan's theorem on the other hand tells us that a projective vector bundle is locally trivial, provided it is isomorphic to a projective subbundle 
of a finite-dimensional trivial vector bundle. In general, however, a projective vector bundle needs not be locally trivial, even if the fibers are finite-dimensional, as the following example shows

3.6. EXAmple. Let $V$ be the vector space $C_{c}(\mathrm{~N})$ of functions with compact support from $\mathrm{N}$ into $\mathrm{C}$ (the vector space of finite $\mathrm{C}$-sequences) with the natural inner product, and let $e_{n}$ be the sequence that has 1 as $n$th coordinate, and otherwise 0 . Then $V$ is a trivial LCS-space. Consider the trivial bundle $\zeta$ $=[0,1] \times V$ and corresponding free $C([0,1])$-module $C([0,1], V)$. Define the function $\theta:(0,1] \rightarrow[0,1]$ by

$$
\theta(x)=\frac{\pi}{2}(x(n+1) n-(n+1)), \quad x \in\left(\frac{1}{n+1}, \frac{1}{n}\right], \quad n \in N
$$

and for each $x \in(0,1]$ define the one dimensional subspace

$$
W_{x}=\operatorname{span}\left\{e_{n} \cos \theta(x)+e_{n+1} \sin \theta(x)\right\}, \quad x \in\left(\frac{1}{n+1}, \frac{1}{n}\right], \quad n \in N
$$

and let the projection $p: \zeta \rightarrow \zeta$ be defined by the fiberwise orthogonal projection onto $W_{x}$ for $x \in(0,1]$ and 0 on the fiber $p_{\zeta}^{-1}(0)$. If $s$ is a section of $\zeta$, $p s$ is also a continuous section, because $p s$ is 0 on a neighborhood of 0 . Thus $p$ induces a well-defined projection $p: C([0,1], V) \rightarrow C([0,1], V)$, and $\operatorname{Im} p$ is a projective bundle (projective $C(X)$-module) that is not locally trivial (then the fiber dimension would be constant by [19]).

We will call an algebra $A$ a trivial algebra in case $A$ has a basis of mutually orthogonal idempotents. Note that $A$ is trivial if and only if $A \cong \mathrm{C}^{(n)}$ for some cardinal $n$ (and therefore separable by corollary 2.16), and that any trivial algebra $A$ equipped with the trivial LCS-topology becomes a locally $m$-convex topological algebra.

We will say that an algebra bundle (a bundle of topological algebras, according to [6], definition 1.5) is fiberwise trivial if each fiber is isomorphic to a trivial algebra with trivial LCS-topology, and completely trivial if $\xi \cong X \times$ $A$ for some trivial algebra $A$ with trivial LCS-topology. It is locally completely trivial if every $x \in X$ has a neighborhood $U$ such that $\xi \mid U$ is completely trivial. We now easily see

3.7. Proposition. Let $X$ be a compact space. The section functor $\Gamma$ is a category equivalence between the category of fiberwise trivial, projective algebra bundles over $X$ and the category of projective $C(X)$-algebras $A$, for which $A / A M$ is a trivial algebra for every maximal ideal $M \subseteq C(X)$.

We further have 
3.8. Proposition. Let $\xi$ be a projective algebra bundle. Then $\xi$ is (locally) completely trivial if and only if $\xi$ (locally) has a basis of mutually orthogonal idempotents.

We now establish a relation between covering spaces and algebra bundles.

3.9. Proposition. Let $X$ be a space. The category of covering spaces over $X$ and the category of locally completely trivial algebra bundles over $X$ are equivalent.

Proof. Let $Y$ be a trivial covering space over $X$, i.e., $Y=X \times D$ for some discrete space $D$. Then $C_{c}(D)=C^{(D)}$ is a trivial algebra, which can be equipped with the trivial LCS topology. To $Y$ we can then associate the completely trivial algebra bundle $X \times C_{c}(D)$. On the other hand, let $\xi=X \times$ $A$ be a completely trivial algebra bundle. The maximal ideal space of $A$ is some discrete space $D$, and to $\xi$ we can then associate the discrete covering space $X \times D$. This describes a category equivalence between trivial covering spaces and completely trivial algebra bundles. Because the automorphism group of $C_{c}(D)$ is naturally isomorphic to the automorphism group of $D$ (the permutation group of $D$ ), this category equivalence can be extended to locally trivial bundles (compare [4], chapter 5, in particular theorem 3.2).

By a regular covering space we will understand a covering space, where the group of automorphisms acts transitively on the fibers. If we consider instead the associated algebra bundle, it is clear that this corresponds to the fact that the group of automorphisms acts transitively on the orthogonal idempotent basis in each point, and we will accordingly denote such an algebra bundle a regular algebra bundle.

\section{Separable algebras and covering spaces}

We now come to the main paragraph of this exposition, where we will investigate the relation between covering spaces of a space $X$ and separable algebras over the ring $C(X)$. In this paragraph $X$ will always denote a compact (Hausdorff) space and $C(X)$ the ring of continuous complex-valued functions on $X$. Our objective is to prove the following theorem

4.1. Theorem. For every locally connected space $X$ there exists a categoryequivalence between the category of regular covering spaces of $X$ and the category of Galois extensions of $C(X)$.

According to proposition 3.9 we can alternatively prove a category equivalence between Galois extensions and regular, locally completely trivial algebra bundles, and this is the strategy we will pursue. 
It is possible to develop a Galois theory of separable algebras, including a Galois group, whereby the fundamental group of $X$ can be found from $C(X)$. However, this development is beyond the scope of this paper, and we will just mention a single corollary to theorem 4.1

4.2. Corollary. Let $X$ be connected and locally connected. Then $X$ is simply connected if and only if $C(X)$ is separably closed.

First we will prove the easy implication of theorem 4,1.

4.3. Proposition. Let $\xi$ be a locally completely trivial algebra bundle over $X$. Then $\Gamma(\xi)$ is a projective, separable algebra over $C(X)$.

Proof. According to theorem 3,5 and proposition 3.7, $\Gamma(\xi)$ is a projective algebra over $C(X)$, and we are to show that $\Gamma(\xi)$ is separable. We know from proposition 3.8 that for every $x \in X$ there exists a neighborhood $U_{x}$ so that $\xi \mid U_{x}$ has an orthogonal, idempotent basis. As $X$ is compact there exists a finite subcovering $\left\{U_{1}, \ldots, U_{n}\right\}$ of $\left\{U_{x}\right\}$ which we shrink to an open covering $\left\{V_{i}\right\}$ satisfying $\bar{V}_{i} \subseteq U_{i}$. We let $\left\{\eta_{i}\right\}$ be a partition of unity subordinated to $\left\{V_{i}\right\}$ and $\left\{e_{\alpha}^{i}\right\}_{\alpha \in A_{i}}$ be the idempotent basis for $\xi \mid U_{i}$. As $\bar{V}_{i}$ is closed, each of the sections $e_{\alpha}^{i} \mid \bar{V}_{i}$ can by proposition 3.3 be extended to a section defined on all of $X$. We will denote these extensions also $e_{\alpha}^{i}$.

Now let $s \in \Gamma(\xi)$ be an arbitrary section. As $\left\{e_{\alpha}^{i} \mid \bar{V}\right\}_{\alpha \in A_{i}}$ is a basis for $\xi \mid \bar{V}_{i}$, the restriction $s \mid \bar{V}_{i}$ can be written as a unique finite sum

$$
s \mid \bar{V}_{i}=\sum_{\alpha \in A_{i}} a_{\alpha}^{i} e_{\alpha}^{i}, \quad a_{\alpha}^{i} \in C\left(\bar{V}_{i}\right)
$$

that is, $a_{\alpha}^{i} \equiv 0$ for all but finitely many $\alpha \in A_{i}$. Once again the functions $a_{\alpha}^{i}$ can be extended (continuously) to all of $X$, and again we will use the notation $a_{\alpha}^{i}$ for these extensions.

With these definitions we then have

$$
s=\sum_{i=1}^{n} \sum_{\alpha \in A_{i}} \eta_{i} a_{\alpha}^{i} e_{\alpha}^{i}
$$

and we have a well-defined map $\varphi: \Gamma(\xi) \rightarrow \Gamma(\xi) \otimes \Gamma(\xi)$ by

$$
\varphi(s)=\sum_{i=1}^{n} \sum_{\alpha \in A_{i}} \eta_{i} a_{\alpha}^{i}\left(e_{\alpha}^{i} \otimes e_{\alpha}^{i}\right)
$$

for every $s \in \Gamma(\xi)$. If $\mu: \Gamma(\xi) \otimes \Gamma(\xi) \rightarrow \Gamma(\xi)$ is the augmentation of $\Gamma(\xi)$, a direct calculation using that $e_{\alpha}^{i}$ is idempotent on $\bar{V}_{i} \supseteq \operatorname{supp} \eta_{i}$ then shows that $\mu \varphi(s)=s$. It is further clear that $\varphi$ is a $C(X)$-module homomorphism, 
and we are to show that it is as well a $\Gamma(\xi) \otimes \Gamma(\xi)$-module homomorphism. Thus, let $t_{1}, t_{2} \in \Gamma(\xi)$. We can write

$$
\begin{array}{ll}
t_{1}=\sum_{i=1}^{n} \sum_{\alpha \in A_{i}} \eta_{i} b_{\alpha}^{i} e_{\alpha}^{i}, \quad b_{\alpha}^{i} \in C(X) \\
t_{2}=\sum_{i=1}^{n} \sum_{\alpha \in A_{i}} \eta_{i} c_{\alpha}^{i} e_{\alpha}^{i}, \quad c_{\alpha}^{i} \in C(X)
\end{array}
$$

Then a simple calculation shows

$$
\varphi(s) \cdot\left(t_{1} \otimes t_{2}\right)=\sum_{i=1}^{n} \sum_{\alpha \in A_{i}} \eta_{i}^{3} a_{\alpha}^{i} b_{\alpha}^{i} c_{\alpha}^{i}\left(e_{\alpha}^{i} \otimes e_{\alpha}^{i}\right)=\varphi\left(s \cdot\left(t_{1} \otimes t_{2}\right)\right)
$$

where we have used that $\left\{e_{\alpha}^{i}\right\}$ is a set of (mutually) orthogonal idempotents on supp $\eta_{i}$. Thus $\varphi$ splits $\mu$ as a $\Gamma(\xi) \otimes \Gamma(\xi)$-module homomorphism, and $\Gamma(\xi)$ is separable.

It remains to prove that if $\xi$ is regular, then $\Gamma(\xi)$ is normal. Let $m \in \mathscr{M}(\Gamma(\xi))$. For every point $x \in X$ we let $\left\{e_{\alpha}^{x}\right\}_{a \in \mathscr{A}_{x}}$ be the orthogonal idempotent basis of $\xi_{x}$. One now sees that for every $e_{\alpha}^{x}$

$$
m\left(e_{\alpha}^{x}\right)=k_{\alpha}^{x} e_{\alpha}^{x}
$$

where $k_{\alpha}^{x} \in \mathrm{C}$. As $\Gamma$ is a functor, every automorphism of $\xi$ induces an automorphism of $\Gamma(\xi)$. For every $\alpha, \beta \in \mathscr{A}_{x}$ there is a $g \in$ Aut $\xi$, such that $g\left(e_{\beta}^{x}\right)=e_{\alpha}^{x}$, since $\xi$ is regular. Then

$$
g m g^{-1}\left(e_{\alpha}^{x}\right)=g m\left(e_{\beta}^{x}\right)=g\left(k_{\beta}^{x} e_{\beta}^{x}\right)=k_{\beta}^{x} e_{\alpha}^{x}
$$

if $m \in \operatorname{Fix}_{\mathrm{Aut} \Gamma(\xi)} \mathscr{M}(\Gamma(\xi))$ we must then have $k_{\beta}^{x}=k_{\alpha}^{x}$ for every $\alpha, \beta \in \mathscr{A}_{x}$ hence $m=f \cdot 1_{\mathscr{M}(A)}$, where $f: X \rightarrow \mathrm{C}$ is some function. The local triviality of $\xi$ ensures us that $f$ must be continuous, so $m \in C(X) \cdot 1_{\mathscr{M}(A)}$. Thus $\Gamma(\xi)$ is normal.

The rest of the paper will be concerned with proving the other implication of theorem 4.1. Thus, assume $A$ is a projective, separable commutative $C(X)$ algebra. In the first part of the proof we will not require $A$ to be normal. Since $A / M A$ is a separable $C(X) / M$-algebra for every maximal ideal $M \subseteq C(X)$ (corollary 2.6), and since $C(X) / M \cong \mathrm{C}$ is separably closed, $A / M A$ is a trivial algebra (corollary 2.16). Thus, by proposition 3.7 there corresponds to $A$ a fiberwise trivial projective algebra bundle, which we will denote $\xi[A]$ (we will regard $\xi$ as a functor), that is, for every point $x \in X$, the fiber $\xi[A]_{x}$ has an orthogonal idempotent basis. What we have to show is that $\xi$ is locally trivial, thus, that the orthogonal idempotent basis for $\xi[A]_{x}$ 
can be extended to a idempotent basis of some neighborhood of $x$. The first major step in this direction is the following proposition

4.4. Proposition. Let $A$ be a projective, separable commutative $C(X)$-algebra and let $\xi=\xi[A]$. For every $x \in X$ and every idempotent $e \in \xi_{x}$ there exists a neighborhood $U$ of $x$, so that e can be extended to an idempotent section over $U$.

For the proof of the proposition we will need a couple of lemmas. First note, that if $\xi$ is a projective vector bundle, then we can imbed $\xi$ as a closed projective subbundle of some trivial vector bundle $\zeta=\left(X \times V, p_{\zeta}, X\right)$. Thus, if $s \in \Gamma(\xi)$ is a section, $\sigma_{\zeta}$ is a continuous function from $X$ to $V$. If $B \subseteq \Gamma(\xi)$ is a finitely generated submodule or if $B$ is contained in a finitely generated submodule, $\sigma_{\zeta}(B)$ is a finite dimensional subspace of $V$. The topology on $\sigma_{\zeta}(B)$ is given by some norm $\|\cdot\|$. Let $B_{x}=\{s(x) \mid s \in B\} \subseteq \xi_{x}$. Then $B_{x}$ is a subspace of $\xi_{x}$, and for $v \in B_{x}$ we can define the norm by $\|v\|=\left\|\sigma_{\zeta} v\right\|$. If $v \in B_{x}$ and $u \in B_{y}$ where not necessarily $x=y$, we let

$$
\|u-v\|=\left\|\sigma_{\zeta} u-\sigma_{\zeta} v\right\|
$$

We can now formulate

4.5. Lemma. Let $A$ be a projective, separable, commutative $C(X)$-algebra and let $B \subseteq A$ be a subalgebra contained in a finitely generated $C(X)$-submodule. For every $x \in X$ let $E_{x}$ be the set of idempotents in $B_{x}$ and $Q_{x}$ the base of orthogonal idempotents of $\xi[A]_{x}$. Then

1) span $E_{x}=B_{x}$.

2) $\left|E_{x}\right|$ (the number of elements in $E_{x}$ ) is bounded on $X$.

3) $\left|\left\{q \in Q_{x} \mid q B_{x} \neq 0\right\}\right|$ is bounded on $X$.

4) $\left\{\|e\| \mid e \in E_{x}, x \in X\right\}$ is bounded from above and from below.

$5) \inf \left\{\left\|e_{1}-e_{2}\right\| \mid e_{1}, e_{2} \in E_{x}, e_{1} \neq e_{2}, x \in X\right\}>0$.

Proof. 1) This is a trivial consequence of the trivial structure of $\xi_{\mathrm{x}}$.

3) First note that $\operatorname{dim} \xi_{x} B_{x}=\left|\left\{q \in Q_{x} \mid q B_{x} \neq 0\right\}\right|$. By theorem 2.8, $A B$ is contained in a finitely generated submodule of $A$. As noted before this lemma, this means that $\operatorname{dim}(A B)_{x}=\operatorname{dim} \xi_{x} B_{x}$ is bounded, hence 3) follows. 2) is now a trivial consequence of 3 ).

4) Assume that $\left\{\|e\| \mid e \in E_{x}, x \in X\right\}$ is unbounded. Then we can find a sequence $\left\{x_{n}\right\} \subseteq X\left(x_{n} \neq x_{m}\right.$ for $\left.n \neq m\right)$ with associated idempotents $e\left(x_{n}\right) \in E_{x_{n}}$, such that $\left\|e\left(x_{n}\right)\right\|>n$. Since the unit sphere $S=\left\{x \in \sigma_{\zeta}(B) \mid\right.$ $\|x\|=1\}$ is compact, 1st countable, $\left\{x_{n}\right\}$ has an (infinite) subsequence (which we also denote $\left\{x_{n}\right\}$ ), such that $\frac{\sigma_{\zeta} e\left(x_{n}\right)}{\left\|\sigma_{\zeta} e\left(x_{n}\right)\right\|} \in S$ converges to some 
point $e$. Now suppose $x \in X$ is a cluster point of $\left\{x_{n}\right\}$. Then $\left\{\frac{e\left(x_{n}\right)}{\left\|e\left(x_{n}\right)\right\|}\right\}$ has one and only one cluster point $c_{x} \in \xi_{x}$. For in the trivial vector bundle $\zeta$ asociated with $\xi$, the only cluster point of $\left\{e\left(x_{n}\right)\right\}$ in $\zeta_{x}$ is $\sigma_{\zeta}^{-1}(e) \cap \zeta_{x}$, and as $\xi$ is embedded in $\zeta$ as a closed subset, by lemma 3.2, this cluster point is in $\xi$. Now define a section $s: \overline{\left\{x_{n}\right\}} \rightarrow \xi$ by

$$
\begin{array}{ll}
s(x)=c_{x} & \text { if } x \text { is a clusterpoint of }\left\{x_{n}\right\} \\
s\left(x_{i}\right)=\frac{e\left(x_{i}\right)}{\left\|e\left(x_{i}\right)\right\|} & \text { otherwise }
\end{array}
$$

Then $s$ is continuous. For, let $x$ be a cluster point of $\left\{x_{n}\right\}$ and let $U \times V \subseteq \zeta$ be a neighborhood of $s(x)$. Since $\sigma_{\zeta} s\left(x_{i}\right)$ converges to $\sigma_{\zeta} s(x)$, we may choose some neighborhood $\tilde{U} \subseteq U$ of $x$, such that $\sigma_{\zeta} s\left(x_{i}\right) \in V$ for all $x_{i} \in \tilde{U}$. Then $s\left(\tilde{U} \cap \overline{\left\{x_{n}\right\}}\right) \subseteq U \times V$, since this is certainly true for any non-cluster point of $\tilde{U} \cap \overline{\left\{x_{n}\right\}}$ and if $y$ is a cluster point, then $\sigma_{\zeta} s(y)=\sigma_{\zeta} s(x)$, hence $\sigma_{\zeta} s(y) \in V$. By proposition $3.3 s$ can be extended to a continuous section on all of $X$.

As $X$ is compact, $s$ is bounded, that is, for all $x \in X,\|s(x)\| \leq k$ for some $k \in \mathrm{R}$. Then

$(*)\left\|s^{2}\left(x_{n}\right)\right\|=\|\| e\left(x_{n}\right)\left\|^{-2} e\left(x_{n}\right)\right\|=\|\| e\left(x_{n}\right)\left\|^{-1} s\left(x_{n}\right)\right\|=\left\|e\left(x_{n}\right)\right\|^{-1}\left\|s\left(x_{n}\right)\right\| \leq \frac{k}{n}$

Since $X$ is compact, there is at least one cluster point $x$ of $\left\{x_{n}\right\}$. By $(*)$ we must have $\left\|s^{2}(x)\right\|=0$, since $s^{2}$ is a continuous section. But by the definition $s(x) \neq 0$, a contradiction.

The proof that $\left\{\|e\| \mid e \in E_{x}, x \in X\right\}$ is bounded from below is similar, except that we assume $\left\|e\left(x_{n}\right)\right\|<\frac{1}{n}$ and then get the contradiction $\left\|s^{2}(x)\right\|=\infty$ for any cluster point of $\left\{x_{n}\right\}$.

5) The proof of 5) is a slight modification of the proof of 4 ).

The next lemma is a generalized form of Vandermonde's lemma, the proof of which we will leave as a simple exercise in algebra

4.6. Lemma (Generalized Vandermonde lemma). Let $P_{j}=\left\{p_{i j}\right\}_{i}=1, . ., n$, $j=1, \ldots, n$ be a family of polynomials. Then

$$
\left|\begin{array}{ccc}
p_{11}\left(x_{1}\right) & \cdots & p_{1 n}\left(x_{n}\right) \\
\vdots & \ddots & \vdots \\
p_{n 1}\left(x_{1}\right) & \cdots & p_{n n}\left(x_{n}\right)
\end{array}\right|=p\left(x_{1}, \ldots, x_{n}\right) \prod_{\substack{i>j \\
P_{i}=P_{j}}}\left(x_{i}-x_{j}\right)
$$

where $p$ is some polynomial in $n$ indeterminates.

The next lemma essentially states that if, in $\mathrm{C}^{n}$ considered as a trivial C- 
algebra, $\left\|x^{i+1}-x^{i}\right\|$ is small for all $i \leq n$, then $x$ is close to an idempotent, independently of what vector space norm we chose on $\mathrm{C}^{n}$.

4.7. Lemma. For each $n \in \mathrm{N}$ there exists a constant $M_{n}$, such that for every $n$ points $a_{1}, \ldots, a_{n} \in \mathrm{C}$ there exist $\delta_{1}, \ldots, \delta_{n} \in\{0,1\}$ satisfying: if $k_{1}, \ldots, k_{n} \in \mathrm{C}$ satisfy

$$
\left|\sum_{i=1}^{n} k_{i}\left(a_{i}^{m+1}-a_{i}^{m}\right)\right| \leq \epsilon \quad m=1 \ldots n
$$

then

$$
\left|\sum_{i=1}^{n} k_{i}\left(a_{i}-\delta_{i}\right)\right| \leq M_{n} \epsilon
$$

The important thing here is to notice that $M_{n}$ does only depend on $n$ and the $\delta$ 's do only depend on the $a$ 's, not on the $k$ 's.

Proof. First we see that we may assume that $a_{i} \neq 0, a_{i} \neq 1$ and $a_{i} \neq a_{j}$ for all $i, j$. For if $a_{i}=0$ or 1 we can put $\delta_{i}=0$ or 1 and reduce the problem to a problem in $n-1$ dimensions, and if $a_{i}=a_{j}$ we can put $\tilde{a}_{i}=a_{i}=a_{j}$ and $\tilde{k}=k_{i}+k_{j}$ and solve the problem for $a_{i}, \ldots, \tilde{a}_{i}, \ldots, a_{j-1}, a_{j+1}, \ldots, a_{n}$ and $k_{1}, \ldots, \tilde{k}, \ldots, k_{j-1}, k_{j+1}, \ldots, k_{n}$.

So, assume $a_{i} \neq 0, a_{i} \neq 1$ and $a_{i} \neq a_{j}$ for all $i, j$. Let

$$
\epsilon_{m}=\sum_{i=1}^{n} k_{i}\left(a_{i}^{m+1}-a_{i}^{m}\right)=\sum_{i=1}^{n} k_{i} a_{i}^{m}\left(a_{i}-1\right) \quad m=1 \ldots n
$$

Then, by assumption, $\left|\epsilon_{m}\right| \leq \epsilon$. The equations $(*)$ are a linear system in the $k_{i}$ 's, which can be solved by Cramer's rule:

$$
\begin{aligned}
k_{i} & =\frac{\left|\begin{array}{ccccccc}
a_{1}\left(a_{1}-1\right) & \cdots & a_{i-1}\left(a_{i-1}-1\right) & \epsilon_{1} & a_{i+1}\left(a_{i+1}-1\right) & \cdots & a_{n}\left(a_{n}-1\right) \\
\vdots & \ddots & \vdots & \vdots & \vdots & \ddots & \vdots \\
a_{1}^{n}\left(a_{1}-1\right) & \cdots & a_{i-1}^{n}\left(a_{i-1}-1\right) & \epsilon_{n} & a_{i+1}^{n}\left(a_{i+1}-1\right) & \cdots & a_{n}^{n}\left(a_{n}-1\right)
\end{array}\right|}{D} \\
= & \sum_{j=1}^{n} \epsilon_{j} D_{j i}
\end{aligned}
$$

where $D_{j i}$ is the complement of the jith coefficient of the system, and $D$ is the determinant of the system, which we recognize as a Vandermonde determinant: 


$$
\begin{aligned}
D & =\left|\begin{array}{ccc}
a_{1}\left(a_{1}-1\right) & \cdots & a_{n}\left(a_{n}-1\right) \\
\vdots & \ddots & \vdots \\
a_{1}^{n}\left(a_{1}-1\right) & \cdots & a_{n}^{n}\left(a_{n}-1\right)
\end{array}\right| \\
& =\left(\prod_{j>i}\left(a_{j}-a_{i}\right)\right)\left(\prod_{i=1}^{n} a_{i}\right)\left(\prod_{i=1}^{n}\left(a_{i}-1\right)\right)
\end{aligned}
$$

We now have

$$
\begin{aligned}
\sum_{i=1}^{n} k_{i}\left(a_{i}-\delta_{i}\right) & =\sum_{i=1}^{n} \frac{\sum_{j=1}^{n} \epsilon_{j} D_{j i}}{D}\left(a_{i}-\delta_{i}\right) \\
& =\sum_{j=1}^{n} \epsilon_{j} f_{j}\left(a_{1}, \ldots, a_{n}\right)
\end{aligned}
$$

where, letting $\tilde{\delta}_{i}=1-\delta_{i}$

$$
\begin{aligned}
f_{j}\left(a_{1}, \ldots, a_{n}\right) & =D^{-1} \sum_{i=1}^{n} D_{j i}\left(a_{i}-\delta_{i}\right) \\
& =D^{-1}\left(\prod_{i=1}^{n}\left(a_{i}-\delta_{i}\right)\right)\left|\begin{array}{ccc}
a_{1}-\tilde{\delta}_{1} & \cdots & a_{n}-\tilde{\delta}_{n} \\
\vdots & \ddots & \vdots \\
a_{1}^{j-2}\left(a_{1}-\tilde{\delta}_{1}\right) & \cdots & a_{n}^{j-2}\left(a_{n}-\tilde{\delta}_{n}\right) \\
1 & \cdots & 1 \\
a_{1}^{j}\left(a_{1}-\tilde{\delta}_{1}\right) & \cdots & a_{n}^{j}\left(a_{n}-\tilde{\delta}_{n}\right) \\
\vdots & \ddots & \vdots \\
a_{1}^{n-1}\left(a_{1}-\tilde{\delta}_{1}\right) & \cdots & a_{n}^{n-1}\left(a_{n}-\tilde{\delta}_{n}\right)
\end{array}\right|
\end{aligned}
$$

The last $n \times n$ determinant is of the type mentioned in the generalized Vandermonde lemma. Here the polynomials (in the $a$ 's) in two columns are identical if and only if $\tilde{\delta}_{i}=\tilde{\delta}_{j}$, that is $\delta_{i}=\delta_{j}$. Thus, by the generalized Vandermonde's lemma 


$$
\begin{aligned}
f_{j}\left(a_{1}, \ldots, a_{n}\right) & =D^{-1}\left(\prod_{i=1}^{n}\left(a_{i}-\delta_{i}\right)\right)\left(\prod_{i<k, \delta_{i}=\delta_{k}}\left(a_{k}-a_{i}\right)\right) p_{j}\left(a_{1}, \ldots, a_{n}\right) \\
& =\frac{\left(\prod_{i=1}^{n}\left(a_{i}-\delta_{i}\right)\right)\left(\prod_{i<k, \delta_{i}=\delta_{k}}\left(a_{k}-a_{i}\right)\right) p_{j}\left(a_{1}, \ldots, a_{n}\right)}{\left(\prod_{i<k}\left(a_{k}-a_{i}\right)\right)\left(\prod_{i=1}^{n} a_{i}\right)\left(\prod_{i=1}^{n}\left(a_{i}-1\right)\right)} \\
& =\frac{p_{j}\left(a_{1}, \ldots, a_{n}\right)}{\left(\prod_{i<k, \delta_{i} \neq \delta_{k}}\left(a_{k}-a_{i}\right)\right)\left(\prod_{i=1}^{n}\left(a_{i}-\tilde{\delta}_{i}\right)\right)}
\end{aligned}
$$

Notice that the degree of $a_{i}$ in $p_{j}$ is equal to the number of elements in the set $\left\{\delta_{k} \mid \delta_{k} \neq \delta_{i}\right\}$.

We will now show how one can choose $\delta_{i}, i=1 . . n$, as functions of $\left(a_{1}, \ldots, a_{n}\right)$ in such a way that all $f_{j}, j=1 . . n$ are bounded as functions of the $\left(a_{1}, \ldots, a_{n}\right)$. That will prove the lemma.

We chose $\delta_{i}=0$ if in the set $\left\{a_{1}, \ldots, a_{n}\right\}$ there exist $a_{n_{1}}, \ldots, a_{n_{k}}\left(n_{j}\right.$ depending on $i)$ satisfying,

$$
\left\{\begin{aligned}
\left|a_{i}-a_{n_{k}}\right| & \leq \frac{1}{2 n} \\
\left|a_{n_{j}}-a_{n_{j-1}}\right| & \leq \frac{1}{2 n} \quad j=2 \ldots k \\
\left|a_{n_{1}}\right| & \leq \frac{1}{2 n}
\end{aligned}\right.
$$

Otherwise we put $\delta_{i}=1$. With this choice of the $\delta_{i}$ 's it follows that

$$
\begin{aligned}
\left|a_{i}-\tilde{\delta}_{i}\right| & \geq \frac{1}{2} \\
\left|a_{i}-a_{j}\right| & \leq \frac{1}{2 n} \Rightarrow \delta_{i}=\delta_{j} \\
\left|a_{i}\right| & \geq \frac{1}{2} \Rightarrow \delta_{i}=1
\end{aligned}
$$

Now, for convenience of exposition, suppose that the indexing is so that, 


$$
\begin{array}{r}
\delta_{1}, \ldots, \delta_{m}=1 \\
\delta_{m+1}, \ldots, \delta_{n}=0 \\
\left|a_{1}\right|, \ldots,\left|a_{p}\right|>1 \\
\left|a_{p+1}\right|, \ldots,\left|a_{n}\right| \leq 1
\end{array}
$$

where necessarily $p \leq m \leq n$, by the choice of the $\delta_{i}$ 's. Using (1) we then have the estimate

$$
\begin{aligned}
f_{j}\left(a_{1}, \ldots, a_{n}\right) & =\frac{\left|p_{j}\left(a_{1}, \ldots, a_{n}\right)\right|}{\left.\mid \prod_{i<j, \delta \neq \delta_{k}}\left(a_{k}-a_{i}\right)\right)\left(\prod_{i=1}^{n}\left(a_{i}-\tilde{\delta}_{i}\right)\right) \mid} \\
& \leq \frac{\left|p_{j}\left(a_{1}, \ldots, a_{n}\right)\right|}{\left|\prod_{i \leq p, k>p}\left(a_{j}-a_{i}\right)\right|\left(\frac{1}{2 n}\right)^{p(n-p) \mid}\left|\prod_{i=1}^{p}\left(a_{i}-\tilde{\delta}_{i}\right)\right|\left(\frac{1}{2}\right)^{n-p}} \\
& =\frac{\left|p_{j}\left(a_{1}, \ldots, a_{n}\right)\right|}{\left|\prod_{i \leq p, k>p}\left(a_{j}-a_{i}\right)\right|\left|\prod_{i=1}^{p}\left(a_{i}-\tilde{\delta}_{i}\right)\right|}(2 n)^{p(n-p)} 2^{n-p} \\
& =\frac{\left|\tilde{p}_{j}\left(a_{1}^{-1}, \ldots, a_{p}^{-1}, a_{p+1}, \ldots, a_{n}\right)\right|}{\left|\prod_{i \leq p, k>p}\left(a_{k} a_{i}^{-1}-1\right)\right|}(2 n)^{p(n-p)} 2^{n-p}
\end{aligned}
$$

Where $\tilde{p}_{j}$ is the polynomial obtained from $p_{j}$ by dividing by $a_{i}^{n-p}, i \leq p$. Since the degree of $a_{i}, i \leq p$, in $p_{j}$ is $n-m \leq n-p, \tilde{p}_{j}(\cdot)$ is in fact a polynomial in $a_{1}^{-1}, \ldots, a_{p}^{-1}, a_{p+1}, \ldots, a_{n}$ (i.e., not a Laurent polynomial). Now we get from (1) and (2)

$$
\left|a_{k} a_{i}^{-1}-1\right| \geq \max \left\{\left|\frac{1}{2 n a_{k}}\right|, 1-\frac{1}{\left|a_{k}\right|}\right\} \geq \frac{1}{2 n+1}, \quad i \leq p, k>p
$$

Hence

$$
f_{j}\left(a_{1}, \ldots, a_{n}\right) \leq\left|\tilde{p}_{j}\left(a_{1}^{-1}, \ldots, a_{p}^{-1}, a_{p+1}, \ldots, a_{n}\right)\right|(2 n(2 n+1))^{p(n-p)} 2^{n-p}
$$

Since $\tilde{p}_{j}$ is a polynomial on a bounded set, $f_{j}$ is bounded for the combination given in (2). As there are finitely many such combinations, $f_{j}$ is bounded for all values of $\left(a_{1}, \ldots, a_{n}\right)$, proving the lemma.

4.8. Lemma. Let $B \subseteq A$ be a subalgebra contained in a finitely generated submodule of the projective separable $C(X)$-algebra $A$, and let $e_{x} \in B_{x}$ be an 
idempotent. For every $\epsilon>0$ there exists a neighborhood $U$ of $x$ such that for each $y \in U$ there exists an idempotent $e_{y} \in B_{y}$ with $\left\|e_{y}-e_{x}\right\|<\epsilon$.

Proof. Suppose, $\sigma_{\xi} B$ is spanned by $\left\{b_{1}, \ldots, b_{N}\right\}$, and consider $b_{1}, \ldots, b_{N}$ also as constant sections. We may assume that the norm is the max-norm with respect to $b_{1}, \ldots, b_{N}$. Let $a \in B$ be a section through $e_{x}$. As $a$ is continuous, there exists a neighborhood $V$ of $x$, such that $\left\|e_{x}-a(y)\right\|<\frac{\epsilon}{2}$ for $y \in V$. Since $e_{x}$ is an idempotent, $a(x)^{i}=a(x)$ for all $i \in \mathrm{N}$, and as $a^{i}$ is continuous, there exists a neighborhood $W$ of $x$, such that

$$
y \in W \Longrightarrow\left\|a(y)^{i+1}-a(y)^{i}\right\|<\frac{\epsilon}{2 M_{N}} \quad i=1 . . N
$$

where $M_{N}$ is the constant obtained in lemma 4.7. Now fix a $y \in W$. Suppose the dimension of $B_{y}$ is $n \leq N$. Then $B_{y}$ has a basis of $n$ orthogonal idempotents $\left\{e_{1}, \ldots, e_{n}\right\}$, and

$$
e_{i}=\sum_{j=1}^{N} k_{i j} b_{j}(y) \quad i=1 \ldots n
$$

for some constants $k_{i j}$. Now let $a_{j}$ be the coordinate of $a(y)$ with respect to $e_{j}$. Then for all $i=1 . . N$ we have

$$
\begin{gathered}
\left\|a(y)^{i+1}-a(y)^{i}\right\|=\left\|\sum_{j=1}^{n}\left(a_{j}^{i+1}-a_{j}^{i}\right) e_{j}\right\| \\
=\left\|\sum_{j=1}^{n} \sum_{m=1}^{N}\left(a_{j}^{i+1}-a_{j}^{i}\right) k_{j m} b_{m}(y)\right\| \leq \frac{\epsilon}{2 M_{N}}
\end{gathered}
$$

or

$$
\forall m=1 \ldots N:\left|\sum_{j=1}^{n}\left(a_{j}^{i+1}-a_{j}^{i}\right) k_{j m}\right| \leq \frac{\epsilon}{2 M_{n}}
$$

Now, by lemma 4.7 there exist $\delta_{1}, \ldots, \delta_{n} \in\{0,1\}$ (depending solely on the $a_{j}$ 's) such that $\forall m=1 \ldots N:\left|\sum_{j=1}^{n}\left(a_{j}-\delta_{j}\right) k_{j m}\right| \leq \frac{\epsilon}{2}$. Then

$$
\left\|a(y)-\sum_{j=1}^{n} \delta_{j} e_{j}\right\|=\left\|\sum_{j=1}^{n} \sum_{m=1}^{N}\left(a_{j}-\delta_{j}\right) k_{j m} b_{m}(y)\right\| \leq \frac{\epsilon}{2}
$$


Now put $e_{y}=\sum_{j=1}^{n} \delta_{j} e_{j}$. Then $e_{y}$ is an idempotent and with $U=V \cap W$, the triangle inequality gives the conclusion of the lemma.

Its now simple to prove proposition 4.4

Proof of Proposition 4.4. Let $s$ be a section through $e$, that is $s(x)=e$, and put $B=s A$, the ideal generated by $s$. By theorem 2.8, $B$ is contained in a finitely generated submodule of $A$, and thus lemma 4.5 and lemma 4.8 apply, and we will use the notation therefrom. Put

$$
\epsilon=\frac{1}{3} \inf \left\{\left\|e_{1}-e_{2}\right\| \mid e_{1}, e_{2} \in E_{x} \cup\{0\}, e_{1} \neq e_{2}, x \in X\right\}
$$

Then $\epsilon>0$, by lemma 4.5. By lemma 4.8 there exist a neighborhood $U$ of $x$, such that for each $y \in U$ there exists an idempotent $e_{y} \in B_{y}$ with $\left\|e_{y}-e_{x}\right\|<\epsilon$. By the choice of $\epsilon, e_{y}$ is the unique idempotent, that satisfies $\left\|e-e_{x}\right\|<\epsilon$. Thus, we have a well-defined idempotent section $e: U \rightarrow \xi$ by $e(y)=e_{y}$, provided we can prove that $e$ is continuous at $y \in U$. Now, given $\delta>0$, there exist a neighborhood $U_{\delta}$ of $y$, such that for each $z \in U_{\delta}$ there exists $\tilde{e}_{z} \in B_{z}$ with $\left\|\tilde{e}_{z}-e_{y}\right\|<\delta$. Since we may assume $\delta<\epsilon,\left\|e_{x}-\tilde{e}_{z}\right\|<2 \epsilon$, and by the choice of $\epsilon$, there is only one such $\tilde{e}_{z}: e_{z}=e(z)$. Hence, $\|e(z)-e(y)\|<\delta$ on $U_{\delta}$, proving continuity.

The proof of proposition 4.4 gives us an extension of an arbitrary idempotent $e \in \xi_{x}$ to an idempotent section over some neighborhood of $x$, by choosing some arbitrary (non-idempotent) section through $e$. Thus, this extension depends on which section we chose and generally there may be more than one idempotent extension of $e$. However, over connected sets the extension is unique:

4.9. Proposition. Let $e \in \xi_{x}$ be an idempotent and let $U$ be a connected set containing $x$. Then there exists at most one idempotent section over $\bar{U}$ through e.

Proof. Suppose $s_{1}, s_{2}: \bar{U} \rightarrow \xi$ are two different idempotent sections through $e$. By theorem 2.8, the algebra $B$ generated by (the extensions of) $s_{1}$ and $s_{2}$ is finitely generated. Thus, lemma 4.5 applies. The function $n: U \rightarrow \mathrm{R}$ defined by $n(x)=\left\|s_{1}(x)-s_{2}(x)\right\|$ is a continuous function. Since $e=s_{1}(x)=$ $s_{2}(x), n$ assumes the value 0 , and since $s_{1} \neq s_{2}$ it also assumes some value $t$ different from zero. Since $U$ is connected and $n$ continuous it must assume any value between 0 and $t$. But this contradicts lemma 4.5.4) and 5).

Over connected sets, more can be said about idempotent sections. The rank of an idempotent $e$ in a (complex) commutative algebra $A$ is the dimension of $e A$. Now 
4.10. Proposition. Let $e: S \rightarrow \xi[A]$ be an idempotent section over some subset $S \subset X$. If $U$ is a connected set such that $\bar{U} \subset S$, the rank of $e \mid U$ is constant.

Proof. Denote the extension of $e \mid \bar{U}$ to all of $X$ also $e$. By theorem 2.8, $e A$ is contained in a finitely generated submodule of $A$, so $\sigma_{\zeta}(e A)$ is a finite dimensional space on which we as usual may assume the topology is given by some norm $\|\cdot\|$.

The rank of $e \mid U$ is has a maximum, $N$. We will show that the set

$$
R_{N}=\{x \in U \mid \operatorname{rank} e(x)=N\}
$$

is both open and closed. Since it is not empty, it must be all of $U$, showing the theorem.

It is easy to show that $R_{N}$ is open. If $x \in R_{N}$, there exists $N$ (rank one) idempotents $e_{1}^{x}, \ldots, e_{N}^{x} \in[e A]_{x}$, such that $e(x)=e_{1}^{x}+\cdots+e_{N}^{x}$. By lemma 4.5, there exists a constant $q>0$, such that for all $y \in X$ and all $e_{1}, e_{2} \in[e A]_{y}$, $e_{1} \neq e_{2}$ we have $\left\|e_{1}-e_{2}\right\| \geq q$. Then by continuity and lemma 4.8 there exists a neighborhood $W$ of $x$ such that for all $y \in W,\|e(x)-e(y)\| \leq \frac{q}{4}$ and such that there exist idempotents $e_{i}^{y} \in[e A]_{y}$ with

$$
\left\|e_{i}^{x}-e_{i}^{y}\right\| \leq \frac{q}{4 N}
$$

Since

$$
\begin{aligned}
\left\|e(y)-\sum_{i=1}^{N} e_{i}^{y}\right\| & =\left\|(e(y)-e(x))-\left(\sum_{i=1}^{N}\left[\left(e_{i}^{y}-e_{i}^{x}\right)+e_{i}^{x}\right]-e(x)\right)\right\| \\
& \leq \frac{q}{4}+N \frac{q}{4 N}=\frac{q}{2}
\end{aligned}
$$

we have

$$
e(y)=\sum_{i=1}^{N} e_{i}^{y}
$$

and since

$$
\left\|e_{i}^{y}\right\|=\left\|e_{i}^{x}+\left(e_{i}^{y}-e_{i}^{x}\right)\right\| \geq q-\frac{q}{4 N}>0
$$

the rank of $e(y)$ is at least $N$, i.e., precisely $N$.

On the other hand, let $x$ be a cluster point of $R_{N}$. By lemma 4.5, the norms of the idempotents in $e A$ are bounded by some constant $K$. Now for every $\delta>0$ there exists a neighborhood $U_{\delta}$ of $x$, such that for all $a \in e A$ with $\|a(x)\| \leq K$ 


$$
\left\|a^{m}(y)-a^{m}(x)\right\| \leq \delta \quad y \in U_{\delta} \quad m=1 \ldots N+1
$$

Now by lemma 4.5, there exist a constant $q>0$, such that for all $y \in X$ and all $e_{1}, e_{2} \in B_{y}, e_{1} \neq e_{2}$, we have $\left\|e_{1}-e_{2}\right\| \geq q$. Put

$$
\delta=\frac{q}{2(1+2 N M+N)},
$$

where $M$ is the constant from lemma 4.7.

Let $\tilde{W} \subset U$ be a neighborhood of $x$, such that $\|e(x)-e(y)\| \leq \delta$ for $y \in \tilde{W}$ and let $W=\tilde{W} \cap U_{\delta}$. By hypothesis there exists a point $z \in W$ such that $e(z)$ has rank $N$, that is $e(z)=e_{1}^{z}+\cdots e_{N}^{z}$. There exists some $\phi_{i} \in e A$, so that $\phi_{i}(z)=e_{i}^{z}$. Then

$$
\begin{aligned}
\left\|\phi_{i}(x)^{m+1}-\phi_{i}(x)^{m}\right\| & =\|\left(\phi_{i}(x)^{m+1}-\phi_{i}(z)^{m+1}\right) \\
& +\left(\phi_{i}(z)^{m+1}-\phi_{i}(z)^{m}\right)+\left(\phi_{i}(z)^{m}-\phi_{i}(x)^{m}\right) \| \leq 2 \delta
\end{aligned}
$$

because $\phi_{i}(z)$ is an idempotent and $z \in U_{\delta}$. Now we prove exactly in the same way as in lemma 4.8 that there exist idempotents $e_{i}^{x} \in B_{x}, i=1 \ldots N$ such that

$$
\left\|\phi_{i}(x)-e_{i}^{x}\right\| \leq 2 \delta M
$$

Since

$$
\left\|e_{i}^{x}\right\|=\left\|\left(e_{i}^{x}-\phi_{i}(x)\right)+\left(\phi_{i}(x)-\phi_{i}(z)\right)+\phi_{i}(z)\right\| \geq q-2 \delta M-\delta>0
$$

we get $e_{i}^{x} \neq 0$. We now have

$$
\begin{aligned}
& \left\|e(x)-\sum_{i=1}^{N} e_{i}^{x}\right\| \\
& =\left\|(e(x)-e(z))+\left(e(z)-\sum_{i=1}^{N}\left(e_{i}^{x}-\phi_{i}(x)\right)+\left(\phi_{i}(x)-\phi_{i}(z)\right)+\phi_{i}(z)\right)\right\| \\
& \leq \delta+0+2 \delta N M+N \delta \leq \frac{q}{2}
\end{aligned}
$$

It follows that

$$
e(x)=\sum_{i=1}^{N} e_{i}^{x}
$$

hence the rank of $e(x)$ is $N$.

Now, let $x$ be an arbitrary point of $X$. Since $A$ is faithful algebra, $A M_{x} \neq$ $A$ (where $M_{x}$ is the maximal ideal of functions vanishing at $x$ ), hence $\{0\} \neq$ $A / A M_{x} \cong \xi_{x}$. Thus, there exist some rank one idempotent $e_{x} \in \xi_{x}$. By pro- 
position 4.4, this idempotent can be extended to some idempotent section $e$ over a neighborhood $V$ of $x$. Since $X$ is assumed locally connected (and compact), there exist some connected neighborhood $U$ of $x$ with $\bar{U} \subseteq V$. By proposition 3.3,e $\mid \bar{U}$ can be extended to a section over all of $X$. We denote this extension also $e$. Our objective is now to show that normality of $A$ ensures that the set

$$
\{g(e)|U| g \in \text { Aut } A\}
$$

is an orthogonal idempotent basis of $\xi \mid U$. By proposition 3.8 this then shows theorem 4.1.

First we will shrink $U$ slightly: let $r$ be a function which is 1 on a connected neighborhood $W$ of $x$ and vanishes on a neighborhood of $X \backslash U$ and consider the functions $g(r e)$. What we actually will show is that

$$
\{g(r e)|W| g \in \text { Aut } A\}
$$

is an orthogonal idempotent basis of $\xi \mid W$.

Since $e_{x}$ is a rank one idempotent, $g\left(e_{x}\right)$ is a rank one idempotent for any $g \in$ Aut $A$. Thus, for arbitrary $g_{1}, g_{2} \in$ Aut $A$, either $g_{1}\left(e_{x}\right)=g_{2}\left(e_{x}\right)$ or $g_{1}\left(e_{x}\right) g_{2}\left(e_{x}\right)=0$. Since $U$ is connected, then either $g_{1}(e)\left|U=g_{2}(e)\right| U$ or $\left(g_{1}(e) \mid U\right)\left(g_{2}(e) \mid U\right)=0$, by proposition 4.9. Hence, (by axiom of choice) there exists a subset $G \subseteq$ Aut $A$ such that

$$
\begin{aligned}
& g_{1}, g_{2} \in G, g_{i} \neq g_{2} \Rightarrow g_{1}(r e) g_{2}(r e)=0 \\
& \forall g \in \text { Aut } A \exists \tilde{g} \in G: g(r e)=\tilde{g}(r e)
\end{aligned}
$$

Notice, that this implies that the set $\{g(r e)[x] \mid g \in G\}$ is linearly independent in $\xi_{x}$ for every $x \in W$.

We can now state

4.11. Lemma. For any $a \in A$ there exist at most finitely many $g \in G$, such that $g(r e) a \neq 0$.

Proof. Assume there exists an infinite set $H \subseteq G$ such that $g(r e) a \neq 0$ for $g \in H$. Note however, that in each point $x \in U$ there are only finitely many $g \in G$ such that $g(r e)[x] a[x] \neq 0$, since the $g(r e)[x]$ are linearly independent (or zero). Thus, there must exist infinitely many points $\left\{x_{g}\right\}_{g \in H}$ such that $g(r e)\left[x_{g}\right] a\left[x_{g}\right] \neq 0$, and by choosing some subset of $H$, we may assume that $x_{g} \neq x_{h}$ for $g \neq h$ and that $H$ is countable. For each $x_{g}$ choose an idempotent $e_{g} \in(a A)_{x_{g}}$ such that $g(r e)\left[x_{g}\right] e_{g} \neq 0$. This is possible since $g(r e)\left[x_{g}\right] a\left[x_{g}\right] \neq 0$.

Now, since by theorem $2.8 \mathrm{aA}$ is contained in a finitely generated submodule of $A$, lemma 4.5 tells us that the idempotents of $a A$ lies in some compact subset of $\xi$. Thus, the infinite set $\left\{e_{g}\right\}_{g \in H}$ has at least one cluster 
point $c$. By choosing a subset of $H$, we may then assume that $\sigma_{\xi} e_{g}$ has precisely one cluster point, $\sigma_{\xi} c$, since $\left\{\sigma_{\xi} e_{g}\right\}$ is in a compact, first countable space. Note that $p_{\xi}(c)$ is a cluster point of $\left\{x_{g}\right\}_{g \in H}$ and that $p_{\xi}(c) \in U$ (as $r$ vanishes on a neighborhood of $X \backslash U$ ). We will argue that $c$ is an idempotent. Since $\sigma_{\xi} e_{g}$ has precisely one cluster point, we then can define a section $s: \overline{\left\{x_{g}\right\}_{g \in H}} \rightarrow \xi$ in same way that we did in the proof of lemma 4.5 and extend this section to all of $X$. Then in every neighborhood of $p_{\xi}(c)$ there is some $x_{g}$ where $s^{2}\left(x_{g}\right)=e_{g}^{2}=e_{g}=s\left(x_{g}\right)$. Since $s^{2}$ is a continuous section, $c^{2}=$ $s^{2}\left(p_{\xi}(c)\right)=s\left(p_{\xi}(c)\right)=c$.

By proposition 4.4, the idempotent $c$ can be extended to an idempotent section $f$ over some neighborhood $V \subseteq U$, which we may assume connected. There exist at most finitely many $g \in H$, such that $g(e)\left[p_{\xi}(c)\right] c \neq 0$, and since $V$ is connected, these are the only elements of $H$, such that $(g(e) \mid V)(f \mid V) \neq$ 0 . We remove these from $H$. Then $\left\{e_{g}\right\}$ still has a cluster point in $c$. Now consider the algebra generated by $a$ and $f$. As this is finitely generated, lemma 4.5 applies. Since $f$ is continuous, there exists for any $\epsilon>0$ a neighborhood $U_{\epsilon} \subseteq V$ of $p_{\xi}(c)$, such that $\|f(y)-c\|<\epsilon$ for $y \in U_{\epsilon}$, and there exists some $x_{g}>U_{\epsilon}$ with associated $e_{g}$, such that $\left\|e_{g}-c\right\|<\epsilon$. Thus $\left\|f\left(x_{g}\right)-e_{g}\right\|$ $<2 \epsilon$. Since $f\left(x_{g}\right)$ and $e_{g}$ are in the same fiber and are both idempotents, $\left\|f\left(x_{g}\right)-e_{g}\right\|<2 \epsilon$ contradicts lemma 4.5 , except of course if $f\left(x_{g}\right)=e_{g}$. But this is not the case, since $g(e)\left[x_{g}\right] e_{g} \neq 0$, while $g(e)\left[x_{g}\right] f\left(x_{g}\right)=0$, since we have removed points where this is not true from $H$.

Now only the last details remain to prove theorem 4.1. For any $a \in A$, the sum

$$
\sum_{g \in G} g(r e) a
$$

is well-defined, since by lemma 4.11 there are only finitely many non-vanishing terms. Thus, the function $m=\sum_{g \in G} g(r e)$ defined by $m(a)=$ $\sum_{g \in G} g(r e) a$ is well-defined and it is clearly a multiplier of $A$. Furthermore, by definition of $G, g(m)=m$ for all $g \in$ Aut $A$. As $A$ was assumed to be normal, we must have $m \in C(X)$. This means that through every rank one idempotent $f \in \xi_{y}, y \in W$, there is a section $g(e)$. For, assume otherwise. Then $m(s)[y]=0$ for every section $s$ through $f$ (since by proposition 4.10 $g(r e)$ is a rank one idempotent), that is $m(y)=0$ (as a function in $C(X)$ ), while $m(e)[y]=r e[y] \neq 0$, a contradiction. Thus, the set

$$
B_{y}=\{g(r e)[y] \mid g \in G\}
$$

is an orthogonal, idempotent basis in every point $y \in W$. Now, if $s: X \rightarrow \xi$ is a section, $g(r e) s \neq 0$ for at most finitely many $g \in G$, by lemma 4.11 . Thus, 
$s \mid W$ is in the span of finitely many of the idempotent sections $g(r e) \mid W$. By definition, $\{g(r e)|W| g \in$ Aut $A\}$ is then a basis of $\xi \mid W$, proving theorem 4.1 (the regularity of $\xi$ clearly follows from the arguments above).

\section{REFERENCES}

1. L. N. Childs, On covering spaces and Galois extensions, Pacific J. Math. 37 (1971), 29-33.

2. Ian Craw and Susan Ross, Separable algebras over a commutative Banach algebra, Pacific J. Math. 104 (1983), 317-336.

3. F. DeMeyer and E. Ingraham, Separable algebras over commutative rings, Lecture Notes in Math., 1971.

4. J. Dixmier and A. Douady, Champs continus d'espaces Hilbertiens et de C-algèbres, Bull. Soc. Math. France 91 (1963), 227-284.

5. R. Douady, and A. Douady, Algèbre et théories Galoisiennes: 2/théories galoisiennes (Ce$\mathrm{dic} /$ Fernand Nathan, Paris 1979).

6. Gerhard Gierz, Bundles of topological vector spaces and their duality, Lecture Notes in Mathematics 955, 1982.

7. V. L.Hansen, Topological aspects of Galois theory for function rings, Colloq. Math. Soc. Janos Bolyai, 55, 1989.

8. V. L. Hansen and Peter Petersen, Groups, coverings and Galois theory, Canad. J. Math 43 (1991), 1281-1293.

9. J. Horváth, Topological Vector Spaces and Distributions, vol. I, Addison-Wesley 1966.

10. D. Husemoller, Fibre Bundles, Graduate Texts in Math. 20, 1966.

11. N. Jacobson, Structure of Rings, American Math. Soc. Colloquium publications XXXVII, 1964.

12. G. Köthe, Topological Vector Spaces, vol. I, Springer Verlag, Berlin 1969.

13. R. Larsen, An Introduction to the Theory of Multipliers, Springer-Verlag, Berlin 1971.

14. A. R. Magid, Algebraically separable extensions of Banach algebras, Michigan Math. J. 21 (1974), 137-143.

15. A. Mallios, Covering spaces and Galois extensions of topological algebras, preliminary report, Abstracts Amer. Math. Soc. 5 (1) (1984), 143 (:847-46-08).

16. A. Mallios, Galois theory of commutative topological algebras, preliminary report, Abstracts Amer. Math. Soc. 6 (2) (1985), 238 (:857-46-28).

17. A. Mallios, Topological Algebras, Localizations and Extensions, book to appear.

18. R. S. Pierce, Associative Algebras, Graduate Texts in Math. 88, 1982.

19. R. G. Swan, Vector bundles and projective modules, Trans. Amer. Math. Soc. 105 (1962), 264-277.

20. J. L. Taylor, Topological invariants of the maximal ideal space of a Banach algebra, Adv. in Math. 19 (1976), 149-206.

21. J. L. Taylor, A bigger Brauer group, Pacific J. Math. 103 (1982), 163-203.

22. B. Wajnryb, Projective and separable extensions of rings of continuous functions, Bull. Acad. Polon. Sci. Sér. Sci. Math. Astr. Phys. 17 (1969), 269-271.

23. W. R. Zame, Covering spaces and the Galois theory of commutative Banach algebras, J. Funct. Anal. 55 (1984), 151-175.

TRLABS / UNIVERSITY OF GALGARY

280, 3553-31 STREET NW

GALGARY, ALBERTA

CANADA T2L $2 \mathrm{~K} 7$ 


\title{
SOME CHARACTERIZATIONS OF THE PROPERTIES $(\mathrm{DN}) \operatorname{AND}(\widetilde{\Omega})$
}

\author{
LE MAU HAI and NGUYEN VAN KHUE
}

\section{Abstract}

The aim of this paper is to show that

$$
H_{w}(B, F)=H(B, F)
$$

for every $\mathrm{LB}^{\infty}$ - regular compact set $B$ in a Frechet space $E$ if and only if $F$ is a Frechet space having property $(\mathrm{DN})$. At the same time, the equivalence between the existence of a $\mathrm{LB}^{\infty}$ regular compact set $B$ in a Schwartz-Frechet space $E$ with an absolute basis and the property $(\widetilde{\Omega})$ of $E$ is also established here.

\section{Introduction}

Let $E$ be a Frechet space with the topology defined by an increasing system of semi-norms $\left\{\|\|_{k}\right\}$. For each subset $B$ of $E$ we define \|\|$_{B}^{*}: E^{*} \rightarrow[0,+\infty]$ given by

$$
\|u\|_{B}^{*}=\sup \{|u(x)|: x \in B\}
$$

where $u \in E^{*}$, the topological dual space of $E$.

Instead of \|\|$_{U_{q}}^{*}$ we write \|\|$_{q}^{*}$, where

$$
U_{q}=\left\{x \in E:\|x\|_{q} \leq 1\right\}
$$

Now we say that $E$ has the property

$$
\begin{aligned}
& \text { (DN) } \exists p \exists d>0 \forall q \exists k, C>0:\|\|_{q}^{1+d} \leq C\|\|_{k}\|\|_{p}^{d} \\
& (\widetilde{\Omega}) \quad \forall p \exists q, d>0 \forall k \exists C>0:\|\|_{q}^{* 1+d} \leq C\|\|_{k}^{*}\|\|_{p}^{* d} \\
& \left(\mathrm{LB}^{\infty}\right) \quad \forall\left\{\rho_{n}\right\}_{n \in \mathrm{N}} \uparrow \infty \forall p \exists q \forall n_{0} \exists N_{0} \geq n_{0}, C>0 \forall u \in E^{*} \\
& \exists n \in \mathrm{N}: n_{0} \leq n \leq N_{0} \quad\|u\|_{q}^{* 1+\rho_{n}} \leq C\|\quad\|_{n}^{*}\|\|_{p}^{* \rho_{n}}
\end{aligned}
$$

The above properties have been introduced and investigated by Vogt (see [9], [10]). 
Note that the following equivalent form of the property (DN) has been formulated by Zahariuta in [12]

$$
((\mathrm{DN}))_{\mathrm{Z}} \quad \forall p \forall q, d>0 \exists k, C>0:\|\|_{q}^{1+d} \leq C\|\|_{k}\|\|_{p}^{d}
$$

Let $E$ and $F$ be locally convex spaces and let $\Omega \subset E$ be open, $\Omega \neq \emptyset$. $f: \Omega \rightarrow F$ is called Gâteaux - holomorphic if for every $y \in F^{*}$, the topological dual space of $F$, the function $y f: \Omega \rightarrow \mathrm{C}$ is holomorphic. This means that its restriction to each finite dimensional section of $\Omega$ is holomorphic as a function of several complex variables.

A function $f: \Omega \rightarrow F$ is called holomorphic if $f$ is continuous and Gâteaux - holomorphic on $\Omega$.

Now let $B$ be a compact subset in a locally convex space $E$ and $F$ a locally convex space. By the standard notation $H(B, F)$ denotes the space of germs of holomorphic functions on $B$ with values in $F$ with the inductive limit topology.

Recall that $f \in H(B, F)$ if there exists a neighbourhood $V$ of $B$ in $E$ and a holomorphic function $\hat{f}: V \rightarrow F$ whose germ on $B$ is $f$. A $F$-valued function $f$ on $B$ is called weakly holomorphic on $B$ if for every $x^{*} \in F_{\beta}^{*}$, the topological dual space of $F$ equipped with the strong topology $\beta\left(F^{*}, F\right), x^{*} f$ can be extended holomorphically to a neighbourhood of $B$. By $H_{w}(B, F)$ we denote the space of $F$-valued weakly holomorphic functions on $B$.

For details concerning holomorphic functions and germs of holomorphic functions on compact subsets of a locally convex space we refer to the books of Dineen [1] and Noverraz [6].

One of aims of this paper is to find some necessary and sufficient conditions for which

$$
H_{w}(B, F)=H(B, F)
$$

The statement $(\omega)$ has been investigated by several authors. Siciak in [8] and Waelbroeck in [11] have considered this problem for the case, where $\operatorname{dim} E<\infty$ and $F_{\beta}^{*}$ is a Baire space. After that, in [4] N. V. Khue and B. D. Tac have shown that $(\omega)$ holds in the case, where $F_{\beta}^{*}$ is still Baire and either $E$ is a nuclear metric space or $F$ is nuclear. The Baireness of $F_{\beta}^{*}$ plays a very important part in the works of the above authors. However, at present, when $F_{\beta}^{*}$ is not Baire, in particular, $F$ is a Frechet space which is not Banach $(\omega)$ has not been established by any authors.

In the second part of this paper we give a characterization of the property (DN) by showing that $(\omega)$ holds if $F$ is a Frechet space having the property $(\mathrm{DN})$ and $B$ is a $\mathrm{LB}^{\infty}$ - regular compact set in a Frechet space $E$, where a compact set $B$ in a Frechet space $E$ is said to be $\mathrm{LB}^{\infty}-$ regular if $[H(B)]_{\beta}^{*}$ has 
property $\left(\mathrm{LB}^{\infty}\right)$. Next, from the obtained result of the second section the third section is devoted to establishing some characterizations of the property $(\widetilde{\Omega})$ of a Schwartz - Frechet space $E$ with an absolute basis.

In through paper $F_{\text {bor }}^{*}$ denotes the space $F^{*}$ equipped with the bornological topology associated with the topology of $F_{\beta}^{*}$. This is the most strong locally convex topology on $F^{*}$ having the same bounded subsets as the $\beta\left(F^{*}, F\right)$ topology. $\left[F_{\text {bor }}^{*}\right]_{\beta}^{*}$ is equipped with the $\beta\left(F^{* *}, F_{\beta}^{*}\right)$ - topology.

AcKnowledgement. The authors would like to express many thanks to the referees for their helpful remarks and suggestions.

\section{Characterization of (DN)}

The main result of this section is the following

2.1. Theorem. Let $F$ be a Frechet space. Then

$$
H_{w}(B, F)=H(B, F)
$$

holds for every $L B^{\infty}$-regular compact set $B$ in a Frechet space $E$ if and only if $F$ has property (DN).

In order to prove Theorem 2.1 we need some lemmas.

2.2. LemMA. Every $L B^{\infty}$ - regular compact set $B$ in a Frechet space $E$ is a set of uniqueness, i.e. if $f \in H(B)$ and $\left.f\right|_{B}=0$ then $f=0$ on a neighbourhood of $B$ in $E$.

Proof. Let $\left\{V_{n}\right\}$ be a decreasing neighborhood basis of $B$ in $E$. Given $f \in H(B)$ with $\left.f\right|_{B}=0$, choose $p \geq 1$ such that $f \in H^{\infty}\left(V_{p}\right)$. For each $n \geq p$, put

$$
\varepsilon_{n}=\|f\|_{n}=\sup \left\{|f(z)|: z \in V_{n}\right\}
$$

Then $\left\{\varepsilon_{n}\right\} \downarrow 0$. By the hypothesis $[H(B)]_{\beta}^{*}$ has property $\left(\mathrm{LB}^{\infty}\right)$ and employing this with $\left\{\rho_{n}\right\}=\left\{\sqrt{\log \frac{1}{\varepsilon_{n}}}\right\} \uparrow \infty$ we have

$$
\begin{gathered}
\exists q \forall n_{0} \exists N_{0} \geq n_{0}, C_{n_{0}}>0 \forall m>0 \exists k_{m}: n_{0} \leq k_{m} \leq N_{0}: \\
\left\|f^{m}\right\|_{q}^{1+\rho_{k_{m}}} \leq C_{n_{0}}\left\|f^{m}\right\|_{k_{m}}\left\|f^{m}\right\|_{p}^{\rho_{k_{m}}}
\end{gathered}
$$

which yields

$$
\|f\|_{q}^{1+\rho_{k_{m}}} \leq C_{n_{0}}^{\frac{1}{m}}\|f\|_{k_{m}}\|f\|_{p}^{\rho_{k_{m}}}
$$

Choose $n_{0} \leq k \leq N_{0}$ such that 


$$
\text { Card }\left\{m: k_{m}=k\right\}=\infty
$$

Then

$$
\begin{aligned}
\|f\|_{q} & \leq\|f\|_{k}^{\frac{1}{1+\rho_{k}}}\|f\|_{p}^{\frac{\rho_{k}}{1+\rho_{k}}} \\
& \leq\left(\varepsilon_{k}\right)^{\frac{1}{1+\rho_{k}}}\left(\varepsilon_{p}\right)^{\frac{\rho_{k}}{1+\rho_{k}}} \rightarrow 0
\end{aligned}
$$

as $k \rightarrow+\infty$.

Hence $\left.f\right|_{V_{q}}=0$. Lemma 2.2 is proved.

2.3. Lemma. Let $F$ be a Frechet space having property $(\mathrm{DN})$. Then $\left[F_{\mathrm{bor}}^{*}\right]_{\beta}^{*}$ has property (DN).

Proof. Let $\left\{U_{n}\right\}$ be a decreasing neighbourhood basis of $0 \in F$. Since $F$ has property $(\mathrm{DN})$ we have

$$
\exists p \forall q \exists k, C>0:\|\|_{q} \leq r\|\|_{p}+\frac{C}{r}\|\|_{k}
$$

for all $r>0$, or in equivalent form [10]

$$
\exists p \forall q \exists k, C>0: U_{q}^{0} \subseteq r U_{p}^{0}+\frac{C}{r} U_{k}^{0} \quad \text { for all } r>0
$$

For $u \in\left[F_{\text {bor }}^{*}\right]_{\beta}^{*}$ and $r>0$ we have

$$
\begin{aligned}
\|u\|_{q}^{* *} & =\sup \left\{\left|u\left(x^{*}\right)\right|: x^{*} \in U_{q}^{0}\right\} \leq \sup \left\{\left|u\left(x^{*}\right)\right|: x^{*} \in r U_{p}^{0}+\frac{C}{r} U_{k}^{0}\right\} \\
& \leq r \sup \left\{\left|u\left(x^{*}\right)\right|: x^{*} \in U_{p}^{0}\right\}+\frac{C}{r} \sup \left\{\left|u\left(x^{*}\right)\right|: x^{*} \in U_{k}^{0}\right\}= \\
& =r\|u\|_{p}^{* *}+\frac{C}{r}\|u\|_{k}^{* *}
\end{aligned}
$$

Hence $\left[F_{\text {bor }}^{*}\right]_{\beta}^{*}$ has property (DN).

Lemma 2.3 is proved.

Proof OF Theorem 2.1 Sufficiency. It suffices to prove that $H_{w}(B, F) \subset H(B, F)$. Let $f \in H_{w}(B, F)$ and $F$ has property (DN), where $B$ is a LB ${ }^{\infty}$ - regular compact set in a Frechet space $E$. By Lemma $2.2 B$ is a set of uniqueness and, hence, we can consider the linear map $\hat{f}: F_{\text {bor }}^{*} \rightarrow H(B)$ given by

$$
\hat{f}\left(x^{*}\right)=\widehat{x^{*} f}
$$

for $x^{*} \in F_{\mathrm{bor}}^{*}$, where $\widehat{x^{*} f}$ is a holomorphic extension of $x^{*} f$ to some neighbourhood of $B$ in $E$. Still by the uniqueness of $B$ it follows that $\hat{f}$ has closed graph. On the other hand, $F_{\text {bor }}^{*}$ is an inductive limit of Banach spaces, $H(B)$ 
is an (LF) - space so by closed graph theorem of Grothendieck [3] $\hat{f}$ is continuous. Since $\hat{f}$ maps bounded subsets of $F_{\text {bor }}^{*}$ to bounded subsets of $H(B)$ then the dual map $\hat{f}^{\prime}:[H(B)]_{\beta}^{*} \rightarrow\left[F_{\text {bor }}^{*}\right]_{\beta}^{*}$ is also continuous. By the hypothesis $[H(B)]_{\beta}^{*}$ has property $\left(\mathrm{LB}^{\infty}\right)$ and by Lemma $2.3\left[F_{\text {bor }}^{*}\right]_{\beta}^{*}$ has property (DN). From a result of Vogt [9] it follows that there exists a bounded subset $L \subset H(B)$ such that $\hat{f}^{\prime}\left(L^{0}\right)$ is a bounded subset of $\left[F_{\mathrm{bor}}^{*}\right]_{\beta}^{*}$, where $L^{0}$ denotes the polar of $L$ in $[H(B)]_{\beta}^{*}$. Hence $\left(\hat{f}^{\prime}\left(L^{0}\right)\right)^{0}$ is a neighbourhood of $0 \in\left[\left(F_{\mathrm{bor}}^{*}\right)_{\beta}^{*}\right]_{\beta}^{*}$. Put $W=\left(\hat{f}^{\prime}\left(L^{0}\right)\right)^{0} \cap F_{\text {bor }}^{*}$. Then $W$ is a neibourhood of $0 \in F_{\text {bor }}^{*}$. We have

$$
\hat{f}(W) \subset L^{00} \cap H(B)
$$

where $L^{00}$ is the bi-polar of $L$. However $L^{00} \cap H(B)$ is the closure of the absolutely convex envelope of $L$ and, hence, it is a bounded subset of $H(B)$. This shows that $\hat{f}(W)$ is bounded in $H(B)$. By the regularity of $H(B)$ there exists a neighbourhood $U$ of $B$ in $E$ such that $\hat{f}(W)$ is contained and bounded in $H^{\infty}(U)$, the Banach space of bounded holomorphic functions on $U$. From the absorption of $W$ it follows that $\hat{f}\left(F_{\text {bor }}^{*}\right) \subset H^{\infty}(U)$. Now we can define a holomorphic function

$$
g: U \longrightarrow\left[F_{\mathrm{bor}}^{*}\right]^{*}
$$

given by

$$
g(z)\left(x^{*}\right)=\hat{f}\left(x^{*}\right)(z)
$$

for $z \in U, x^{*} \in F_{\text {bor }}^{*}$.

We see that $g(z)\left(x^{*}\right)=\hat{f}\left(x^{*}\right)(z)=f(z)\left(x^{*}\right)$ for every $z \in B, x^{*} \in F^{*}$. This yields $\left.g\right|_{B}=f$ and since $B$ is a set of uniqueness, $g(U) \subset F$.

Necessity. By Vogt [9] it suffices to show that every continuous linear map $T$ from $H(\Delta)$ to $F$ is bounded on a neighbourhood of $0 \in H(\Delta)$, where $\Delta=\{z \in \mathrm{C}:|z|<1\}$. Consider $T^{*}: F_{\beta}^{*} \rightarrow[H(\Delta)]_{\beta}^{*} \cong H(\bar{\Delta})$. Since $T^{*}\left(x^{*}\right) \in$ $H(\bar{\Delta})$ for all $x^{*} \in F_{\beta}^{*}$, we can define a $\operatorname{map} f: \bar{\Delta} \rightarrow\left[F_{\beta}^{*}\right]_{\beta}^{*}$ given by

$$
f(z)\left(x^{*}\right)=\delta_{z}\left(T^{*}\left(x^{*}\right)\right)
$$

for $x^{*} \in F_{\beta}^{*}, z \in \bar{\Delta}$, where $\delta_{z}$ is the Dirac functional defined by $z$

$$
\delta_{z}(\varphi)=\varphi(z) \text { for } \varphi \in H(\bar{\Delta}) .
$$

From the weak continuity of $T^{*}$ and $\delta_{z}$ we infer that $f(z)$ is $\sigma\left(F^{*}, F\right)$-continuous and, hence, $f(z) \in F$. Moreover, $f \in H_{w}(\bar{\Delta}, F)$. Since $\bar{\Delta}$ is $\mathrm{LB}^{\infty}$ regular it follows that $f \in H(\bar{\Delta}, F)$. Thus there exists a neighbourhood $V$ of $\bar{\Delta}$ such that $f \in H^{\infty}(V, F)$. Hence, $B=f(V)$ is bounded in $F$. It is easy to see that $T^{*}$ is bounded on $B^{0}$. Put $C=T^{*}\left(B^{0}\right) \subset[H(\Delta)]_{\beta}^{*}$ and $U=C^{0}$. Then $U$ is a neighbourhood of $0 \in H(\Delta)$ and $T(U) \subset B^{00}$ is bounded in $F$. 
Theorem 2.1 is proved.

\section{Some characterizations of $(\widetilde{\Omega})$}

This section is devoted to give some characterizations of the property $(\widetilde{\Omega})$ on a Schwartz - Frechet space $E$ with an absolute basis.

The following theorem is the main result of this section.

3.1. TheOrem. Let E be a Schwartz-Frechet space with an absolute basis. Then the following are equivalent

(i) There exists a compact set $B$ of uniqueness in $E$ such that $H_{w}(B, F)=H(B, F)$ for all Frechet spaces $F$ having property (DN).

(ii) There exists a compact set $B$ in $E$ such that $[H(B)]_{\beta}^{*}$ has property $\left(\mathrm{LB}^{\infty}\right)$.

(iii) There exists a compact set $B$ in $E$ which is not polar.

(iv) E has the property $(\widetilde{\Omega})$.

\section{PROOF.}

(ii) $\Rightarrow$ (i) by Theorem 2.1 .

Now we give the proof (i) $\Rightarrow$ (iii). The implication (i) $\Rightarrow$ (iii) is obtained from the following proposition

3.2. Proposition Let $B$ be a compact set of uniqueness in a Frechet space $E$ having a Schauder basis and let

$$
H_{w}(B, F)=H(B, F)
$$

for every Frechet space $F \in(\mathrm{DN})$. Then $B$ is not polar.

Proof. Otherwise, assume that $B$ is polar. Choose a plurisubharmonic function $\varphi$ on $E$ such that $\varphi \neq-\infty$ and

$$
\left.\varphi\right|_{B}=-\infty
$$

Consider the Hartogs domain $\Omega_{\varphi}$ given by

$$
\Omega_{\varphi}=\left\{(z, \lambda) \in E \times \mathrm{C}:|\lambda|<e^{-\varphi(z)}\right\}
$$

Since $\varphi$ is plurisubharmonic, $\Omega_{\varphi}$ is pseudoconvex. Because $E$ has a Schauder basis so $\Omega_{\varphi}$ is the domain of a holomorphic function $f$. Write the Hartogs expansion of $f$

$$
f(z, \lambda)=\sum_{n=0}^{\infty} h_{n}(z) \lambda^{n}
$$

where 


$$
h_{n}(z)=\frac{1}{2 \pi i} \int_{|\lambda|=e^{-\varphi(z)-\delta}} \frac{f(z, \lambda)}{\lambda^{n+1}} d \lambda, \text { for } \delta>0 .
$$

By the upper semi-continuity of $\varphi$ it follows that $h_{n}$ is holomorphic on $E$ for all $n \geq 0$. Consider the function $g: B \rightarrow H(\mathrm{C})$, given by $g(z)(\lambda)=f(z, \lambda)$. Let $\mu \in[H(\mathrm{C})]_{\beta}^{*}$ be arbitrary. There exists $r>0$ such that $\mu \in[H(r \bar{\Delta})]_{\beta}^{*}$, where $\bar{\Delta}=\{\lambda \in \mathrm{C}:|\lambda| \leq 1\}$. From the openness of $\Omega_{\varphi}$ it follows that there exists a neighbourhood $V$ of $B$ such that $V \times r \Delta \subset \Omega_{\varphi}$. By the absolute convergence of the series $\sum_{n=0}^{\infty} h_{n}(z) \lambda^{n}$ on $V \times r \Delta$ it follows that $\mu g \in H(V)$ and, hence, $g \in H_{w}(B, H(\mathrm{C}))$. Applying the hypothesis to $F=H(\mathrm{C})$ which has property (DN) we find a neighbourhood $U$ of $B$ in $E$ and a bounded holomorphic function $\hat{g} \in H(U, H(\mathrm{C}))$ which is a holomorphic extension of $g$. We can write

$$
\hat{g}(z, \lambda)=\sum_{n=0}^{+\infty} \hat{g}_{n}(z) \lambda^{n}
$$

where $\hat{g}_{n}(z)$ is holomorphic on $U$ for all $n \geq 0$. Choose a neighbourhood $W$ of $B$ such that $W \subset U$ and $W \times 2 \Delta \subset \Omega_{\varphi}$. Define two holomorphic functions

$$
\begin{aligned}
H: W & \rightarrow H^{\infty}(\Delta) \\
z & \mapsto\left(h_{0}(z), h_{1}(z), \ldots\right) \\
G: W & \rightarrow H^{\infty}(\Delta) \\
z & \mapsto\left(\hat{g}_{0}(z), \hat{g}_{1}(z), \ldots\right)
\end{aligned}
$$

Since $H^{\infty}(\Delta)$ is a Banach space and $\left.H\right|_{B}=\left.G\right|_{B}$, it follows that there exists a neighbourhood $W_{1}$ of $B$ in $\mathrm{W}$ such that $\left.\widehat{g}\right|_{W_{1} \times \Delta}=\left.f\right|_{W_{1} \times \Delta}$. Let $\mathrm{X}$ be a connected component of $W_{1}$. Since $X \times \mathrm{C}$ is connected, $\left.\widehat{g}\right|_{X \times \Delta}=\left.f\right|_{X \times \Delta}$, $X \times \Delta \subset \Omega_{\varphi}$ and $\Omega_{\varphi}$ is the domain of existence of $f$ we have $X \times \mathrm{C} \subset \Omega_{\varphi}$. Hence $\left.\varphi\right|_{X}=-\infty$. This is impossible.

Proposition 3.2 is proved.

The following proposition gives the implication (iii) $\Rightarrow$ (iv).

3.3. Proposition. Let $E$ be a Frechet space. If there exists a non polar compact set in $E$ then $E$ has property $(\widetilde{\Omega})$.

Proof. By a result of Dineen - Meise - Vogt [2, Corollary 8 and Theorem 10].

Finally, the implication (iv) $\Rightarrow$ (ii) is given by the following proposition.

3.4. Proposition. Let E be a Schwartz-Frechet space with an absolute ba- 
sis. If $E$ has the property $(\widetilde{\Omega})$ then there exists a balanced convex compact subset $B$ of $E$ such that $[H(B)]_{\beta}^{*}$ has property $\left(\mathrm{LB}^{\infty}\right)$.

Proof. Let $\left\{e_{j}\right\}_{j \geq 1}$ be an absolute basis for $E$. From the hypothesis, by Vogt [9], there exists a balanced convex compact set $B_{1}$ in $E$ such that

$$
\left(\widetilde{\Omega}_{B_{1}}\right) \quad \forall p \exists q, d>0, C>0:\|\|_{q}^{* 1+d} \leq C\|\|_{B_{1}}^{*}\|\|_{p}^{* d}
$$

On the other hand, since $\left\{e_{j}\right\}_{j \geq 1}$ is an absolute basis it follows that $\left\|e_{j}^{*}\right\|_{B_{1}}^{*} e_{j}$ converges to $0 \in E$. Put

$$
B=\overline{\operatorname{conv}}\left(B_{1} \cup \cup\|\|_{j \geq 1}^{*} \|_{B_{1}}^{*} e_{j}\right)
$$

Now we prove that $[H(B)]_{\beta}^{*}$ has property $\left(\mathrm{LB}^{\infty}\right)$.

In order to prove that $[H(B)]_{\beta}^{*}$ has property $\left(\mathrm{LB}^{\infty}\right)$ by Vogt [9], it suffices to show that every continuous linear map $T:[H(B)]_{\beta}^{*} \longrightarrow H(\mathrm{C})$ is bounded in a neighbourhood of $0 \in[H(B)]_{\beta}^{*}$. Consider the function $f: B \longrightarrow H(\mathrm{C})$ given by

$$
f(x)(\lambda)=T\left(\delta_{x}\right)(\lambda) \text { for } x \in B, \lambda \in \mathrm{C}
$$

where $\delta_{x} \in[H(B)]_{\beta}^{*}$ is the Dirac functional associated with $x$. We claim that $f$ is weakly holomophic, i.e. $\mu f \in H(B)$ for all $\mu \in[H(\mathrm{C})]_{\beta}^{*}$. Indeed, since $E$ is a Schwartz-Frechet space so $[H(B)]_{\beta}^{*}$ is also a Schwartz-Frechet space. Now let $\mu \in[H(\mathrm{C})]_{\beta}^{*}$ then $\mu T \in\left[[H(B)]_{\beta}^{*}\right]_{\beta}^{*}=H(B)$ which gives a holomorphic extension of $\mu f$. For each $s>0$ consider $h^{s}=R^{s} f$, where $R^{s}: H(\mathrm{C}) \longrightarrow H^{\infty}(2 s \Delta)$ is the restriction map and $\Delta=\{\lambda \in \mathrm{C}:|\lambda|<1\}$. Then $h^{s}$ can be extended to a bounded holomorphic function $\widehat{h}^{s}$ on a neighbourhood $V^{s}$ of $B$ in $E$. Take $p \geq 1$ such that $B+U_{p} \subset V^{1}$ and $\left(\widetilde{\Omega}_{B_{1}}\right)$ holds for $E$, where $U_{p}=\left\{x \in E:\|x\|_{p} \leq 1\right\}$. Let $V_{1}=B+U_{p}$ and let $\bar{g}:(B \times \mathrm{C}) \cup\left(V_{1} \times \bar{\Delta}\right) \longrightarrow \mathrm{C}$ be given by

$$
\bar{g}(x, \lambda)= \begin{cases}f(x)(\lambda) & \text { for } x \in B, \lambda \in \mathrm{C} \\ \widehat{h}^{1}(x)(\lambda) & \text { for } x \in V_{1}, \lambda \in \bar{\Delta}\end{cases}
$$

Obviously $\bar{g}$ is separately holomorphic in the sense of Siciak [7]. By $\mathscr{F}$ we denote the family of all finite dimensional subspaces $P$ of $E(B)$, where $E(B)$ denotes the Banach space induced by $B$. Put

$$
\bar{g}_{P}=\left.\bar{g}\right|_{(B \cap P \times \mathrm{C}) \cup\left(V_{1} \cap P \times \bar{\Delta}\right)}
$$

Since $B \cap P$ and $\bar{\Delta}$ are not pluri - polar in $V_{1} \cap P$ and $\mathrm{C}$, respectively, by Nguyen and Zeriahi [5] $\bar{g}_{P}$ is extended uniquely to a holomorphic function $\widehat{g}_{P}$ on $\left(V_{1} \cap P\right) \times \mathrm{C}$. Since $V_{1} \cap E(B)=\cup\left\{V_{1} \cap P: P \in \mathscr{F}\right\}$ the family $\left\{\widehat{g}_{P}: P \in \mathscr{F}\right\}$ defines a Gâteaux holomorphic function $\widehat{g}$ on $\left(V_{1} \cap E(B)\right) \times \mathrm{C}$. 
On the other hand, $\bar{g}$ is holomorphic on $\left\{x \in B:\|x\|_{B}<1\right\} \times \Delta$, by Zorn's theorem $\widehat{g}$ is holomorphic on $\left(V_{1} \cap E(B)\right) \times \mathrm{C}$, where $V_{1} \cap E(B)$ is equipped with the topology of $E(B)$.

Now we prove that $\widehat{g}$ is extended holomorphically to $\widehat{g}_{1}$ on $W \times \mathrm{C}$, a neighbourhood of $B \times \mathrm{C}$ in $E \times \mathrm{C}$ such that $\widehat{g}_{1}(W \times r \Delta)$ is bounded for $r>0$. Let $q \geq p, d>0, C>0$ be chosen such that (1) holds.

Since $B=\overline{\operatorname{conv}}\left(B_{1} \cup \underset{j \geq 1}{\cup}\left\|e_{j}^{*}\right\|_{B_{1}}^{*} e_{j}\right)$ we have

$$
\left\|e_{j}^{*}\right\|_{B_{1}}^{*}\left\|e_{j}\right\|_{B} \leq 1, \text { for } j \geq 1
$$

From the condition (1) we have

$$
\left(\frac{1}{\left\|e_{j}\right\|_{q}}\right)^{1+d} \leq \frac{C}{\left\|e_{j}\right\|_{B}\left\|e_{j}\right\|_{p}^{d}}
$$
that

Now let $\delta=\frac{1}{2}\left(C^{\frac{1}{1+d}} e\right)^{-1}$. Given $r>0, d>0$ we can find $s, D>0$ such

$$
\|\sigma\|_{r}^{1+d} \leq D\|\sigma\|_{s}\|\sigma\|_{1}^{d}
$$

for $\sigma \in H(\mathrm{C})$, where

$$
\|\sigma\|_{k}=\sup \{|\sigma(z)|:|z| \leq k\}
$$

Write the Taylor expansion of $g: V_{1} \cap E(B) \longrightarrow H(\mathrm{C})$, the function associated to $\widehat{g}:\left(V_{1} \cap E(B)\right) \times \mathrm{C} \longrightarrow \mathrm{C}$ at $0 \in E(B)$

$$
g(x)=\sum_{n=0}^{\infty} P_{n} g(x)
$$

where

$$
P_{n} g(x)(\lambda)=\frac{1}{2 \pi i} \int_{|t|=1} \frac{\widehat{g}(t x, \lambda)}{t^{n+1}} d t
$$

for $x \in V_{1} \cap E(B), \lambda \in \mathrm{C}$.

Since $\widehat{h}^{s}$ is holomorphic at $0 \in E$ for every $s>0$ we infer that $P_{n} g(\cdot)(\lambda)$ is continuous on $E$ for every $\lambda$. Let $\widehat{P_{n} g}$ be the symmetric $n$-linear form associated with $P_{n} g$. We have

$$
\begin{aligned}
\sum_{n \geq 0}\left|P_{n} g(x)(\lambda)\right| & \leq \sum_{n \geq 0} \sum_{j_{1}, \cdots, j_{n} \geq 1} \frac{\left|e_{j_{1}}^{*}(x)\right|\left\|e_{j_{1}}\right\|_{q} \cdots\left|e_{j_{n}}^{*}(x)\right|\left\|e_{j_{n}}\right\|_{q}}{\left\|e_{j_{1}}\right\|_{q} \cdots\left\|e_{j_{n}}\right\|_{q}} \\
& \times\left|\widehat{P_{n} g}\left(e_{j_{1}}, \cdots, e_{j_{n}}\right)(\lambda)\right|
\end{aligned}
$$

Using (2), (3) and (4) we get 


$$
\begin{aligned}
\sum_{n \geq 0}\left|P_{n} g(x)(\lambda)\right| \leq \sum_{n \geq 0} \sum_{j_{1}, \cdots, j_{n} \geq 1} \frac{D^{\frac{1}{1+d}} C^{\frac{n}{1+d}}\left|e_{j_{1}}^{*}(x)\right|\left\|e_{j_{1}}\right\|_{q} \cdots\left|e_{j_{n}}^{*}(x)\right|\left\|e_{j_{n}}\right\|_{q}}{\left\|e_{j_{1}}\right\|_{B}^{\frac{1}{1+d}} \cdots\left\|e_{j_{n}}\right\|_{B}^{\frac{1}{1+d}}\left\|e_{j_{1}}\right\|_{p}^{\frac{d}{1+d}} \cdots\left\|e_{j_{n}}\right\|_{p}^{\frac{d}{1+d}}} \\
\quad \times\left\|\widehat{P_{n} g}\left(e_{j_{1}}, \cdots, e_{j_{n}}\right)\right\|_{s}^{\frac{1}{1+d}}\left\|\widehat{P_{n} g}\left(e_{j_{1}}, \cdots, e_{j_{n}}\right)\right\|_{1}^{\frac{d}{1+d}} \\
\leq D^{\frac{1}{1+d}} \sum_{n \geq 0} C^{\frac{n}{1+d}} \frac{n^{n}}{n !}\left\|P_{n} g\right\|_{s, B}^{\frac{1}{1+d}}\left\|P_{n} g\right\|_{1, p}^{\frac{d}{1+d}}\|x\|_{q}^{n} \\
\leq D^{\frac{1}{1+d}}\|g\|_{B \times s \Delta}^{\frac{1}{1+d}}\|g\|_{U_{p} \times \Delta}^{\frac{d}{1+d}} \sum_{n=0}^{\infty} C^{\frac{n}{1+d}} \frac{n^{n}}{n !} \delta^{n}<+\infty
\end{aligned}
$$

for $x \in \delta U_{p}$ and $|\lambda|<r$.

Thus $g$ is extended holomorphically to $\left(\delta U_{q} \times \mathrm{C}\right) \cup\left(V_{1} \times \bar{\Delta}\right)$. By the same argument, as above, $g$ is extended holomorphically to $g_{1}$ on $V_{1} \times \mathrm{C}$. Consider $\widehat{g}_{1}: V_{1} \longrightarrow \dot{H}(\mathrm{C})$ associated with $g_{1}$. By the same above argument it follows that $\widehat{g}_{1}$ is locally bounded. Hence there exists a neighbourhood $W$ of $B$ in $V_{1}$ such that $\widehat{g}_{1}$ is bounded. Define a continuous linear map $S:\left[H^{\infty}(W)\right]^{*} \longrightarrow H(\mathrm{C})$ as

$$
S(\mu)(\lambda)=\mu\left(\widehat{g}_{1}(\cdot, \lambda)\right)
$$

Since (1) holds for $B_{1}$ it holds for $B$. This shows that $B$ is a set of uniqueness and we infer that $\operatorname{span} \delta(B)$ is weakly dense in $[H(B)]_{\beta}^{*}$. Because $[H(B)]_{\beta}^{*}$ is reflexive $\operatorname{span} \delta(B)$ is dense in $[H(B)]_{\beta}^{*}$, where $\delta: B \rightarrow[H(B)]_{\beta}^{*}$ is given by $\delta(x)(\varphi)=\varphi(x), x \in B, \varphi \in H(B)$. Now we have

$$
\begin{aligned}
& T\left(\sum_{j=1}^{m} \lambda_{j} \delta_{z_{j}}\right)(\lambda)=\sum_{j=1}^{m} \lambda_{j} T\left(\delta_{z_{j}}\right)(\lambda)=\sum_{j=1}^{m} \lambda_{j} f\left(z_{j}, \lambda\right) \\
& =\sum_{j=1}^{m} \lambda_{j} \widehat{g}_{1}\left(z_{j}, \lambda\right)=\sum_{j=1}^{m} \lambda_{j} S\left(\delta_{z_{j}}\right)(\lambda)=S\left(\sum_{j=1}^{m} \lambda_{j} \delta_{z_{j}}\right)(\lambda)
\end{aligned}
$$

for $\lambda \in \mathrm{C}$.

Hence $\left.S\right|_{[H(B)]_{\beta}^{*}}=T$ and $[H(B)]_{\beta}^{*} \in\left(\mathrm{LB}^{\infty}\right)$.

Proposition 3.4 is proved.

\section{REFERENCES}

1. S. Dineen, Complex Analysis in Locally Convex Spaces, Math. Stud. 57 (1981).

2. S. Dineen, R. Meise and D. Vogt, Characterization of nuclear Frechet spaces in which every bounded set is polar, B.S.M.F. 112 (1984), 41-68.

3. A. Grothendieck, Produits tensoriels topologiques et espaces nucléaires, Mem. Amer. Math. Soc. 16 (1955).

4. Nguyen Van Khue and Bui Dac Tac, Extending holomorphic maps from compact sets in infinite dimension, Studia. Math. 95 (1990), 263-272. 